Annuaire suisse de politique de développement

$19 \mid 2000$

Pour une politique plus cohérente envers les pays en développement

\title{
7. Coopération avec les pays en développement
}

Valérie Bouloudani, Eléonore D’Achon, Gérard Perroulaz et John-Andri Lys

\section{(2) OpenEdition}

Journals

Édition électronique

URL : http://journals.openedition.org/aspd/854

DOI : 10.4000/aspd.854

ISSN : 1663-9669

Éditeur

Institut de hautes études internationales et du développement

Édition imprimée

Date de publication : 1 avril 2000

Pagination : 293-326

ISSN : 1660-5934

\section{Référence électronique}

Valérie Bouloudani, Eléonore D'Achon, Gérard Perroulaz et John-Andri Lys, « 7. Coopération avec les pays en développement ", Annuaire suisse de politique de développement [En ligne], 19 | 2000, mis en ligne le 19 août 2012, consulté le 08 septembre 2020. URL : http://journals.openedition.org/aspd/854 ; DOI : https://doi.org/10.4000/aspd.854 


\section{COOPÉRATION AVEC LES PAYS EN DÉVELOPPEMENT*}

\section{1. ÉVOLUTION GÉNÉRALE DE L'AIDE PUBLIQUE AU DÉVELOPPEMENT}

Le Comité d'aide au développement (CAD) est un comité spécialisé de l'OCDE chargé de traiter des questions de coopération au développement. Le CAD a pour but d'accroître le volume total des ressources versées aux pays en développement et d'améliorer l'efficacité de la coopération au développement. Cette instance permet, entre autres, aux pays donateurs de fournir des statistiques harmonisées sur l'aide publique au développement (APD), d'examiner périodiquement (tous les trois ans) les programmes d'aide de chacun des membres, d'échanger des expériences et d'élaborer des stratégies communes pour améliorer la qualité de l'aide.

\section{$\square$ Evolution de l'aide publique au développement des pays $d u$ CAD}

En 1998, l'APD versée par les pays membres du CAD aux pays en développement et aux agences multilatérales de développement a atteint 51,5 milliards (chiffre provisoire) contre 48,3 milliards de dollars en 1997 (soit une augmentation de 3,2 milliards de dollars) ${ }^{1}$. En termes réels (compte tenu de l'inflation et des fluctuations des taux de change), l'APD des membres du CAD a progressé de $8,9 \%$. Cette augmentation met fin à une période de cinq années de diminution des apports d'aide. En effet, en raison, notamment, des mesures d'assainissement des finances publiques dans les pays membres du CAD, les budgets de coopération, et plus particulièrement ceux des pays membres du G7, ont connu depuis 1992 une diminution. Ainsi, entre 1992 et 1997, l'APD des pays du CAD, en termes réels, a connu une diminution de $21 \%$. Pour la même période, l'APD des membres du CAD en pourcentage de leurs PNB combinés a chuté de $0,33 \%$ en 1992 à 0,22\% en 1997 (chiffre le plus bas jamais enregistré). Pour le CAD, la remontée des apports d'aide en 1998 est principalement due à des mesures à court terme pour soutenir les pays touchés par la crise asiatique, au calendrier des contributions aux agences multilatérales, ainsi qu'aux engagements fermes de certains pays membres de maintenir ou d'augmenter leurs apports d'aide (par exemple: engagement du Royaume-Uni d'augmenter son aide de $25 \%$ d'ici 2001, engagement du gouvernement allemand de renverser la tendance à la baisse de l'APD allemande).

Deux pays membres du CAD sur trois ont augmenté leurs apports d'aide en termes réels en 1998. Toutefois, seuls quatre pays (Danemark, Norvège, PaysBas, Suède) dépassent l'objectif fixé par les Nations Unies de 0,7\% du PNB. Les Etats-Unis, qui ont augmenté leur aide de 1,3 milliard de dollars en 1998, demeurent le pays qui fournit l'effort le plus faible avec seulement $0,10 \%$ de leur PNB

\footnotetext{
* Parties 7.1, 7.2, 7.4, 7.5 et 7.7: par Valérie Bouloudani, doctorante à l'IUED.

Partie 7.8: par Eléonore d'Achon, diplômée IUED.

Partie 7.3: par Valérie Bouloudani et Eléonore d'Achon.

Partie 7.6: par Gérard Perroulaz, chargé de recherche à l'IUED.

Partie 7.9: par John-Andri Lys, secrétaire général, Commission suisse pour le partenariat scientifique avec les pays en développement, Berne.

1. OCDE, Communiqué de presse, 10.6.99.
} 
consacré à l'APD'2. En consacrant 0,32\% de leur PNB à l'APD en 1998, la Suisse n'a pas dépassé l'effort moyen des pays du CAD (0,4\% du PNB).

Parmi les pays membres du G7 (qui ont contribué ensemble à près des trois quarts de l'APD en 1998), les plus grands apports d'aide, en valeur absolue, provenaient du Japon (10,7 milliards de dollars en 1998), des Etats-Unis (8,1 milliards), de la France (5,9 milliards) et de l'Allemagne (5,6 milliards). Les pays non membres du G7 ont fourni 26\% de l'APD des pays du CAD en 1998 alors que leur part dans le PNB de l'ensemble des pays du CAD n'était que de $13 \%$.

\section{- Evolution de la mise en ouvre}

de la stratégie de partenariat pour le développement

En mai 1996, les pays membres du CAD ont adopté un rapport ${ }^{3}$ dans lequel ils réaffirment que la coopération internationale peut apporter un soutien efficace au développement des pays du Sud. Pour réaliser, d'ici 2015, les objectifs quantitatifs et qualitatifs de développement issus des grandes conférences internationales des Nations Unies tenues dans les années 90, les membres du CAD proposent un nouveau partenariat mondial en faveur du développement et s'engagent à:

- manifester la volonté de conclure des engagements réciproques avec les partenaires en développement, appuyés par des ressources suffisantes;

๖ améliorer la coordination de l'aide en appui aux stratégies locales de développement;

- s'efforcer de garantir la cohérence entre les politiques d'aide et les politiques suivies dans d'autres domaines qui ont une incidence pour les pays en développement.

[D ASTM 1997, objectifs du CAD (pp. 142-143).

Ce rapport, qui émane d'une déclaration intitulée «Vers un partenariat pour le développement dans le nouveau contexte mondial», adoptée en 1995, représente la stratégie à suivre pour un partenariat efficace au service du développement. Les quatre grands thèmes autour desquels s'articule ce rapport constituent désormais l'ossature du programme de travail du CAD: servir les objectifs de développement convenus à l'échelon international; renforcer les partenariats et améliorer le fonctionnement de l'aide; mobiliser des ressources au service du développement et en assurer le suivi; veiller à la cohérence des politiques affectant les pays en développement.

Selon l'édition 1999 du rapport sur la coopération pour le développement du $\mathrm{CAD}^{4}$, la poursuite de la stratégie pour améliorer l'efficacité du partenariat au service du développement est plus que jamais nécessaire dans un contexte marqué par la crise financière des économies émergentes et la diminution des apports de ressources aux pays en développement. Dans ce rapport, James H. Michel,

2. Il faut toutefois rappeler que, depuis 1997, l'aide à Israël ne peut plus être comptabilisée dans l'APD. Ainsi, deux tiers de l'importante baisse du volume de l'APD des Etats-Unis enregistrée entre 1996 et 1997 s'explique par le retrait d'Israël de la liste des pays et territoires en développement du CAD.

3. OCDE, Le rôle de la coopération pour le développement à l'aube du XXIe siècle, OCDE, Paris, mai 1996

4. OCDE, Coopération pour le développement. Rapport 1998: Efforts et politiques des membres du Comité d'aide au développement, OCDE, Paris, 1999 (parution annuelle en début d'année sur l'aide des membres du CAD de l'année précédente). 
président du CAD, dont le mandat a pris fin en janvier $1999^{5}$, passe en revue les progrès accomplis dans la mise en œuvre de cette stratégie, dont les plus notables concernent les initiatives prises par les pays membres du CAD pour renforcer la coordination du système d'aide international sur la base du principe du partenariat.

Dans le prolongement du Forum des partenaires au développement, organisé par le CAD en janvier 1998, les hauts fonctionnaires ont entériné une liste d'actions à engager pour renforcer les partenariats en faveur du développement et simplifier et harmoniser les procédures utilisées par les donateurs. Cette liste émane des résultats de l'examen, réalisé sous l'égide du CAD, du fonctionnement du système international d'aide au Mali, des résultats de vastes consultations dans les pays en développement, et des études de cas relatives notamment aux problèmes d'appropriation par les bénéficiaires de l'aide et aux procédures des donateurs. Les principales dispositions concrètes envisagées pour améliorer l'efficacité du partenariat pour le développement sont les suivantes:

- aider les pays partenaires à se doter des capacités nécessaires pour définir euxmêmes leurs propres stratégies et assurer eux-mêmes la coordination de l'aide, notamment en accueillant chez eux les réunions des groupes consultatifs;

- rendre transparents les intérêts respectifs des pays donateurs et des pays bénéficiaires partenaires et rehausser la confiance réciproque par un dialogue permanent;

- harmoniser les procédures administratives et les obligations en matière de notification, qui diffèrent actuellement d'un donateur à un autre, de même que le cycle de programmes afin d'atténuer la charge imposée aux pays partenaires;

- permettre aux pays partenaires de tirer des avantages de l'appel à la concurrence internationale et de l'approvisionnement auprès des fournisseurs locaux par un déliement de l'aide;

- mettre un terme à la prolifération des projets et privilégier l'aide-programme et le soutien budgétaire afin de respecter les priorités stratégiques des pays bénéficiaires en matière de développement;

$\checkmark$ multiplier les exercices conjoints de suivi et évaluation des programmes de développement par les donateurs et les bénéficiaires;

$\checkmark \quad$ veiller à la cohérence des politiques affectant les pays en développement;

」 innover au niveau des méthodes de financement afin que l'APD joue un rôle de levier pour attirer d'autres formes d'investissement;

๖ poursuivre les mesures d'allégement de la dette.

Pour le CAD, les pays membres et les organismes multilatéraux fournissent sans conteste des efforts pour adapter leurs systèmes et leurs pratiques aux principes de la stratégie du partenariat pour le développement dans le but d'améliorer concrètement la coordination de l'aide et de faciliter la tâche aux pays en développement. Nombre d'entre eux conduisent actuellement des initiatives pilotes qui vont dans ce sens. Les expériences démontrent que l'application de cette stratégie pose des exigences élevées aussi bien aux pays en développement qu'aux

5. Jean-Claude Faure, haut fonctionnaire français, a succédé à James H. Michel en février 1999. 
pays industrialisés. A terme, le CAD souhaite une application systématique de l'approche fondée sur le partenariat afin que celle-ci devienne la norme dans la coopération internationale au développement.

Dans son «Message concernant la continuation de la coopération technique et de l'aide financière en faveur des pays en développement» du 7 décembre 1998, le Conseil fédéral réaffirme que la Suisse souscrit à la stratégie de politique de développement du CAD et qu'elle continuera à s'engager activement en faveur d'une meilleure coordination des donateurs au sein du CAD. La Direction du développement et de la Coopération (DDC) du Département fédéral des affaires étrangères a d'ailleurs financé en 1999 une rencontre des responsables des questions de développement de l'OCDE afin d'étudier la mise en œuvre de la stratégie de partenariat pour le développement dans les agences de coopération des pays du CAD. En outre, la Suisse poursuivra son rôle de leader dans un programme pilote de promotion du développement participatif et de la bonne gestion des affaires publiques au Bénin.

\subsection{AIDE PUBLIQUE AU DÉVELOPPEMENT FOURNIE PAR LA SUISSE}

\section{$\square$ Organisation et gestion de l'aide au développement}

La politique suisse de coopération au développement repose sur trois piliers:

a la Loi fédérale sur la coopération au développement et l'aide humanitaire internationale (du 19 mars 1976) et son ordonnance d'application (du 12 décembre 1977). La loi définit les objectifs généraux de l'aide, à savoir contribuer en priorité à soutenir les efforts des pays en développement en vue d'améliorer les conditions de vie de leurs populations et permettre à ces pays d'être en mesure d'assurer leur propre développement. Elle précise également les formes que peut revêtir la coopération, dont les principales sont la coopération technique, l'aide financière, les mesures de politique économique et commerciale, et l'aide humanitaire;

- les crédits de programme pluriannuels, présentés dans un message du Conseil fédéral aux Chambres fédérales. Les crédits de programme (ou créditscadres) constituent la base financière de l'aide publique au développement) de la Suisse. Pour chacun des principaux instruments de coopération, les Chambres fédérales doivent approuver des engagements financiers (en principe tous les quatre ans), garantissant à l'administration fédérale une base plus stable pour les activités de coopération. Les messages du Conseil fédéral permettent aussi de faire le bilan des expériences passées et de préciser les grandes orientations de l'aide pour les années à venir. Le Parlement fixe ensuite l'enveloppe globale annuelle des fonds disponibles pour l'aide au développement dans le cadre de l'adoption du budget de la Confédération;

口 les documents déterminant les orientations des relations de la Suisse avec les pays du Sud, comme par exemple les Lignes directrices Nord-Sud.

ASTM 1999, le tableau 19 (p. 223) présente la liste des crédits de programme actuellement en vigueur. (Seul le crédit-cadre en faveur des mesures de politique économique et commerciale n'est plus en vigueur.)

[] ASTM 2000, le dossier du présent Annuaire est consacré aux Lignes directrices Nord-Sud. 
Au niveau fédéral, deux offices, relevant respectivement du Département fédéral des affaires étrangères (DFAE) et du Département fédéral de l'économie (DFE), sont chargés en priorité de la coopération au développement. La Direction du développement et de la coopération (DFAE) englobe les domaines suivants: coopération technique (assistance technique et mise à disposition de fonds et de matériel pour la réalisation de projets de développement) et financière (prise en charge de certains investissements précis) avec les pays en développement; aide humanitaire; et coopération technique avec les pays de l'Est. Elle est en outre responsable de la coordination globale de la coopération au développement. Le Secrétariat d'Etat à l'économie (Seco), du DFE, est chargé quant à lui des mesures de politique économique et commerciale dans le cadre de la coopération au développement ainsi que de la coopération financière avec les pays de l'Europe de l'Est et ceux de la Communauté des Etats indépendants (CEI). Le Seco, né de la fusion entre l'Office des affaires économiques extérieures (OFAEE) et l'Office fédéral du développement économique et de l'emploi (OFDE), est opérationnel depuis le $1^{\text {er }}$ juillet 1999, sous la direction du secrétaire d'Etat David Syz. DDC et Seco assument en commun la responsabilité de l'aide financière multilatérale.

\section{$\square$ Volume de l'aide publique au développement en 1998 et évolution récente}

En 1998, l'aide de la Suisse aux pays en développement s'est élevée à 1537 millions de francs (aide privée et publique). La Confédération a versé 1266,4 millions (soit $82 \%$ de l'aide), les cantons et les communes 20,6 millions et les organisations privées d'entraide 250 millions.

\section{Aide publique au développement fournie par la Confédération}

L'APD versée par plusieurs départements de l'administration fédérale a atteint 1266,4 millions de francs en 1998. La DDC a géré plus de $81 \%$ de l'APD, soit un peu plus d'un milliard de francs. Le Seco a versé environ 152 millions de francs au titre des mesures de politique économique et commerciale. D'autres départements ou offices interviennent dans la coopération au développement mais, ensemble, ils ont géré moins de $6 \%$ de l'APD de la Confédération. Il s'agit du Département fédéral des finances (6,8 millions de francs), du Département de l'intérieur (3 millions de francs) pour les bourses d'études accordées par l'Office fédéral de l'éducation et de la science (OFES), de l'Office fédéral de l'environnement, des forêts et du paysage (OFEFP), etc.

Avec une progression de plus de 60 millions de francs, l'APD fournie par la Confédération a retrouvé son niveau de 1996. Ce résultat est dû principalement à une forte progression en 1998 des dépenses du Seco, qui a mis en place de nouveaux instruments d'appui au secteur privé. Cependant, la Suisse s'éloigne de l'objectif maintes fois affirmé du Conseil fédéral de consacrer $0,4 \%$ du PNB à l'APD (la Suisse n'a jamais souscrit à l'objectif des Nations Unies d'une aide se montant à $0,7 \%$ du PNB). En pourcentage du PNB, l'APD de la Suisse est passée, ces dernières années, de $0,36 \%$ en 1994 à $0,34 \%$ en 1996, pour atteindre $0,32 \%$ en 1997 et en 1998 . Compte tenu de la situation financière de la Confédération et des coupures budgétaires adoptées par le Parlement, les moyens prévus dans le plan financier pour la période 1999-2002 feront baisser, ces prochaines années, l'APD à $0,31 \%$ du PNB, voire à $0,29 \%$ en $2002^{6}$.

6. «Message du Conseil fédéral concernant la continuation de la coopération technique et de l'aide financière en faveur des pays en développement» du 7 décembre 1998, Feuille fédérale (mesage n 98.072). 
Aide publique au développement fournie par les cantons et les communes Conséquence des restrictions budgétaires, l'aide fournie par les cantons et les communes baisse depuis 1992 pour atteindre 20,6 millions de francs en 1998. En six ans, les versements d'aide au développement des cantons et des communes ont chuté de $25 \%$. Il faut néanmoins préciser que ceux-ci avaient doublé entre 1987 et 1992 (28,2 millions de francs en 1992). En 1998, les cantons ont versé environ $61 \%$ de l'aide au développement des collectivités territoriales et locales (13,2 millions de francs). Les aides fournies par les cantons de Zurich et de Genève et par les deux demi-cantons de Bâle représentent plus des deux tiers des versements d'aide cantonale. Il faut toutefois relever les efforts pour la solidarité internationale de la part de certains cantons à capacité financière plus faible, tels que Jura et Neuchâtel. L'aide communale s'élevait à 8,4 millions de francs en 1998. Ce sont les communes des cantons de Genève et Zurich qui ont versé le plus d'aide (respectivement 3,3 et 1,7 million de francs).

Répartition de l'APD selon les principaux instruments de coopération D'une année à l'autre, la répartition de l'APD entre les principaux instruments de coopération demeure assez stable. En 1998, deux tiers de l'APD bilatérale et multilatérale de la Suisse étaient consacrés à la coopération technique et à l'aide financière. L'aide humanitaire et alimentaire représentait $18 \%$ de l'APD. Sans toutefois revenir à son niveau de 1994, la part des mesures de politique économique et commerciale a progressé de 4 points entre 1997 et 1998 (passant de $8 \%$ à $12 \%$ ), avec l'entrée en vigueur en janvier 1997 du nouveau crédit-cadre et la mise en œuvre de nouveaux instruments d'appui au secteur privé.

\section{$\square$ La conditionnalité politique en matière de politique de développement ${ }^{7}$}

Afin d'assurer une plus grande cohérence de la politique extérieure de la Suisse, le Conseil fédéral a décidé, en décembre 1998, qu'il serait désormais compétent pour l'application du principe de la conditionnalité politique. A l'avenir, la décision d'interrompre, totalement ou partiellement, la coopération avec un pays pour des raisons politiques ou en cas de violation grave et systématique des droits humains sera prise par le Conseil fédéral, et non plus par un département fédéral, voire un office fédéral. Confronté à un cas concret, il est prévu que le Conseil fédéral procède à une évaluation de la situation en prenant en considération l'ensemble des relations extérieures de la Suisse avec le pays en question. Les domaines pouvant faire l'objet d'une mesure d'application du principe de la conditionnalité politique sont nombreux : coopération au développement, promotion de la paix ou promotion des droits de l'homme, mais aussi commerce extérieur, garantie contre les risques à l'exportation, etc. L'aide humanitaire échappe à la conditionnalité politique.

Le Conseil fédéral a statué sur les critères et les modalités d'application du principe de la conditionnalité politique. Au nombre des critères déterminant une interruption partielle ou totale de la coopération avec un pays figurent:

- des efforts insuffisants quant à l'application du principe de la bonne gestion des affaires publiques (good governance);

- des violations graves des droits de l'homme;

7. Voir DDC, Promotion des droits de l'homme et coopération au développement. Lignes directrices, DDC, Berne, 1997. 
- la suspension de processus de démocratisation;

- des atteintes importantes à la paix et à sécurité;

- l'absence de volonté d'accepter le retour sur son territoire de ses propres ressortissants.

Ces critères sont surtout observés dans le cadre des relations bilatérales avec les autres Etats et, dans la mesure du possible, dans le cadre multilatéral car l'efficacité du principe de la conditionnalité dépend en fin de compte surtout de la cohérence de la démarche des pays donateurs.

Pour le Conseil fédéral, le principe de la conditionnalité politique ne s'applique pas automatiquement. Les mesures plus positives qu'une suspension de la coopération et le dialogue politique sont privilégiés. L'interruption partielle ou totale de la coopération avec un pays ne doit constituer que le dernier recours et peut s'avérer indispensable pour préserver la crédibilité des objectifs de la politique extérieure suisse. Dans une approche nuancée de l'application de la conditionnalité politique, il est possible de réorienter ou de compléter des programmes ou des projets existants en choisissant d'autres canaux pour les réaliser. Ainsi, l'aide peut être retirée aux organismes gouvernementaux et confiée aux ONG.

Dans le passé, la conditionnalité politique en matière de développement a été appliquée à l'encontre de la Bolivie, du Myanmar, du Népal, du Tchad, de l'Ouganda, du Pérou, du Mozambique, du Kenya, du Rwanda et de Madagascar, ainsi que, dans le cadre de la coopération avec les pays de l'Est, à l'encontre de l'Albanie. Plus récemment, faisant suite au coup d'Etat militaire au Niger, qui a renversé et mis fin aux jours du président Ibrahim Baré Maïnassara en avril 1999, la Suisse a suspendu provisoirement son programme de coopération avec ce pays. Pour ne pas pénaliser injustement les populations, les programmes de coopération avec les acteurs non gouvernementaux ont été maintenus. Ainsi, $80 \%$ de l'aide suisse (environ 8 millions par an) échappe au moratoire. En effet, depuis 1996 (année de l'avant-dernier coup d'Etat), la Suisse a réorienté ses programmes de coopération avec le Niger pour appuyer les communautés de base et les organisations de la société civile dans leurs activités pour le développement. L'interruption de la coopération au développement avec le gouvernement nigérien a touché quatre programmes de la DDC dans les domaines de l'hydraulique, de l'environnement, des pistes et de l'élevage. La préparation de nouveaux programmes avec l'administration de ce pays a également été interrompue. La poursuite des programmes de coopération au développement avec le gouvernement du Niger dépend du rétablissement de l'Etat de droit et à des progrès au niveau du respect des libertés fondamentales dans ce pays.

CD ASTM 1999, «Promotion des droits humains» (pp. 227-228).

\subsection{NOUVEAU CRÉDIT DE PROGRAMME POUR LA COOPÉRATION TECHNIQUE ET L'AIDE FINANCIÈRE}

\section{$\square$ Montant et durée du nouveau crédit de programme}

Les mesures de la coopération suisse au développement reposent notamment sur l'adoption de crédits de programme, que le Parlement approuve en principe tous les quatre ans. Dans l'année passée sous revue, l'actualité a été marquée par 
l'adoption d'un nouveau crédit de programme de 4 milliards de francs pour les années 1999-2002 afin de poursuivre la coopération technique et l'aide financière de la Confédération ${ }^{8}$. Ce nouveau crédit vient remplacer l'ancien crédit de programme de 3,8 milliards de francs, épuisé mi-1999.

Si ce nouveau crédit-cadre de 4 milliards de francs traduit une augmentation nominale de l'aide publique au développement de 200 millions de francs par rapport au précédent crédit de programme, le poids de l'aide suisse par rapport au produit national brut (PNB) se réduit quant à lui toujours plus (voir le sous-chapitre 7.2 ci-dessus).

La coopération technique et l'aide financière représentent les principaux instruments de l'APD suisse. Ceux-ci s'appliquent aussi bien sur le plan bilatéral que multilatéral. Pour ces prochaines années, la coopération suisse continuera à mettre l'accent sur la coopération technique et l'aide financière bilatérale. En effet, par rapport au crédit précédent, le rapport entre la coopération bilatérale et multilatérale demeurera inchangé pour les années 1999-2002: il sera maintenu dans une proportion de deux tiers contre un tiers en ce qui concerne le budget de la DDC.

CD ASTM 1995, sur l'ancien crédit de programme pour la coopération technique et l'aide financière (1995-1999) ainsi que le message y relatif (pp. 128-131).

\section{$\square$ Débats aux Chambres}

Suite à l'approbation par le Conseil des Etats du crédit de programme de 4 milliards de francs pour la coopération technique et l'aide financière en faveur des pays en développement, la Communauté de travail a tenté d'inverser la tendance à la baisse de l'aide au développement en proposant une augmentation du créditcadre à 4,5 milliards de francs. Sur proposition de Remo Gysin (PS/BS), la demande fut donc votée au Conseil national, en concurrence avec la proposition de l'Union démocratique du centre (UDC), qui, à l'opposé, souhaitait une diminution du crédit-cadre à 3,6 milliards. C'est la proposition d'augmentation de 500 millions de francs qui l'a finalement emporté, par 78 voix contre 73 . Toutefois, cette augmentation, étant supérieure à 20 millions, a été soumise au frein aux dépenses, un arrêté fédéral qui requiert que les dépenses excédant un tel montant soient approuvées à la majorité absolue. Or, au moment du vote sur cette clause financière, la proposition d'augmentation du crédit-cadre n'obtint que 92 voix contre 52 . Cette dernière fut donc annulée, et le crédit d'aide au développement repartit au Conseil des Etats. Celui-ci, qui avait donné son accord pour 4 milliards par 32 voix sans opposition, approuva à nouveau la proposition du Conseil fédéral. Finalement, la proposition d'un crédit de 4 milliards de francs en faveur des pays en développement a été votée le 16 juin 1999 au Conseil national, par 126 voix contre 1 .

\section{$\square$ Principes de base de la coopération suisse}

Le «Message du Conseil fédéral concernant la continuation de la coopération technique et de l'aide financière» pour la période 1999-2002 a été une occasion de faire le bilan des actions passées et de donner les grandes orientations pour les années à venir. Ainsi, ce nouveau message annonce la volonté du Conseil fédéral de poursuivre, voire de renforcer, les principes et les tendances amorcés dans le crédit-cadre précédent:

8. Cf. «Message du Conseil fédéral concernant la continuation...» du 7 décembre 1998, p. 143. 
- le financement d'équipements et d'infrastructures constituera de moins en moins la clé de voûte des projets de développement pour les prochaines années. Il s'agira, au contraire, de renforcer et d'encourager les processus d'apprentissage et de changement. Ainsi, plusieurs principes et thèmes transversaux guideront la politique de l'aide suisse: participation et empowerment, lutte contre la pauvreté, développement équilibré hommes-femmes et environnement;

- il s'agira également d'améliorer la coordination des donateurs, de renforcer le dialogue politique avec les gouvernements et institutions partenaires ainsi que de promouvoir un cadre politique (good governance) et des conditions macro-économiques favorables au développement. Dans cette optique, la coopération suisse souhaite renforcer les instruments et approches suivants: lutte contre la corruption; promotion des droits de l'homme et du principe d'Etat de droit, et encouragement aux efforts de décentralisation des pays partenaires. Ces mesures constitueront des enjeux centraux de la coopération au développement pour la période 1999-2002.

\section{$\square$ Coopération bilatérale: axes prioritaires géographiques et thématiques}

La DDC compte poursuivre la tendance amorcée dans le crédit-cadre précédent, qui consiste à engager ses moyens là où ils sont le plus utiles. Il s'agira donc, pour la coopération suisse, de renforcer sa politique de concentration géographique et thématique, cela dans l'optique de pouvoir participer pleinement à la coordination de l'aide au sein des organismes internationaux spécialisés et, dans un contexte de restriction budgétaire, d'éviter une dispersion des moyens financiers.

La politique de concentration géographique se traduira notamment par l'allocation de $70 \%$ du volume financier de la coopération bilatérale à 17 pays de concentration ${ }^{9}$. Au cours de ces dernières années, la DDC a fermé ses bureaux de coordination (BUCO, voir plus loin sous-chapitre 7.5) en Indonésie, en Thaïlande et au Cap-Vert. En 1997, le Vietnam est devenu un pays dit de concentration pour la coopération suisse. Suite aux lenteurs de l'enquête sur l'assassinat d'un coopérant suisse en 1996 et à une mauvaise gestion des affaires publiques à Madagascar, la Suisse a décidé, à la fin de l'année 1998, de réorienter son programme de coopération après un examen approfondi et une visite du directeur de la DDC à Madagascar. Depuis 1996, elle a réduit le volume de son aide. Cinquième pays bénéficiaire de l'aide suisse en 1995 (20,5 millions de francs), Madagascar verra encore son enveloppe chuter pour atteindre environ 7 millions de francs par an dès 2001. La DDC concentre désormais ses activités sur les programmes de lutte contre la pauvreté dans les zones rurales en collaborant exclusivement avec les organisations de la société civile. Le bureau de coordination a été fermé et la coordination de son programme de coopération est maintenant assurée par des ONG. Dans le crédit-cadre de la coopération technique et de l'aide financière pour la période 1999-2002, l'Equateur, qui était un pays de deuxième priorité pour la DDC mais avec un bureau de coordination, devient un nouveau pays de concentration.

9. A savoir: Bénin, Burkina Faso, Mali, Niger et Tchad pour l'Afrique de l'Ouest; Mozambique et Tanzanie pour l'Afrique de l'Est; Bolivie, Equateur, Nicaragua/Région d'Amérique centrale et Pérou pour l'Amérique latine; Bangladesh, Inde, Népal, Pakistan, Bhoutan et Vietnam pour l'Asie. 
Comme l'illustre l'exemple du Mozambique (qui fait l'objet de l'encadré suivant), cette politique de concentration a pour principal objectif d'améliorer la qualité de l'aide apportée par la Suisse.

\section{Le Mozambique: un pays de concentration de la DDC}

Depuis que la DDC a fait du Mozambique un de ses pays de concentration, le programme d'approvisionnement en eau a été étendu et renforcé. Partant d'un appui à la mise en place de systèmes d'approvisionnement en eau à la campagne, les problèmes spécifiques de construction, de fonctionnement et de maintenance des installations d'eau potable en zone urbaine ont été abordés. La Suisse soutient le programme national de formation d'hydrauliciens destinés à travailler dans tout le pays, ainsi que l'administration nationale des eaux dans son effort de décentralisation. En outre, en tant que donateur principal pour le Mozambique, elle a pu jouer un rôle clé dans le développement social de ce pays, et a ainsi réussi à améliorer la coordination parmi les donateurs. La communication qui en est résultée entre gouvernements et donateurs a permis de former la base d'un dialogue politique permanent ainsi que de mettre au point une approche sectorielle intégrée en fonction de laquelle s'orientent le gouvernement et une grande partie des donateurs.

Source: "Message du Conseil fédéral concernant la continuation de la coopération technique et de l'aide financière» du 7 décembre 1998 (pp. 54 et 84).

Le principe de concentration thématique se manifestera, quant à lui, par un choix encore plus sélectif des domaines d'intervention de la DDC. En effet, cette dernière se limitera à trois, voire quatre axes prioritaires thématiques par pays afin d'accroître son niveau de spécialisation. Ces axes prioritaires sont les suivants: développement rural; environnement et utilisation durable des ressources naturelles; Programme mondial pour l'environnement; approvisionnement en eau et assainissement; infrastructure routière et transports; promotion de l'artisanat et de l'industrie, formation professionnelle; système financier; santé et questions démographiques; formation de base et urbanisation.

\section{Un projet de protection de l'environnement: les petites exploitations minières en Bolivie}

En Bolivie, près de $10 \%$ de la population vit de l'extraction minière. Cette activité dégrade considérablement l'environnement et est source de conflits entre mineurs et agriculteurs (pollution de l'eau par les rejets de mines). La Suisse soutient l'application de la législation environnementale bolivienne en collaboration avec des institutions publiques et privées. Ce projet comprend notamment la planification et la mise en œuvre de mesures de protection de l'environnement, la formation du personnel, le soutien à la création de capacités institutionnelles et la collaboration avec de nombreuses organisations nationales et internationales.

Source: «Message du Conseil fédéral concernant la continuation de la coopération technique et de l'aide financière» du 7 décembre 1998 (р. 52).

Parallèlement à sa politique de concentration, la DDC souhaite renforcer l'approche régionale dans le dessein d'étendre au-delà des frontières nationales des projets ayant donné des résultats probants dans les pays de concentration. Cette approche, déjà mise en œuvre en Amérique centrale et dans la région du Mékong, sera élargie à d'autres régions. La coopération suisse au développement compte ainsi contribuer à stimuler les contacts entre pays voisins et à promouvoir la coopération régionale. 


\section{Succès du projet «Postcosecha» au Honduras: une mise en valeur régionale réussie}

Le projet "Postcosecha" a débuté en 1980 au Honduras, où les parasites détruisaient près d'un cinquième des denrées alimentaires après la récolte. Ces pertes ont pu être pratiquement évitées grâce à la mise en place de simples silos en métal permettant aux paysans de stocker les aliments de base. Outre le fait d'améliorer la sécurité alimentaire (près d'un quart de la population cible du Honduras dispose d'un silo pouvant couvrir les besoins de cinq adultes pendant un an) et d'avoir par conséquent des incidences positives sur la santé de nombreuses familles, le projet "Postcosecha" permet aux petits exploitants d'être moins dépendants et de disposer d'un revenu additionnel. En effet, grâce à ce système, les paysans ne sont plus obligés de vendre leurs produits à des conditions défavorables immédiatement après la récolte, pour acheter plus tard les produits dont ils ont besoin à un prix beaucoup plus élevé. Couronné de succès, ce projet a été transposé dans les années 90 au Guatemala, au Nicaragua, au Salvador et au Paraguay.

Source: "Message du Conseil fédéral concernant la continuation de la coopération technique et de l'aide financière» du 7 décembre 1998 (pp. 85-86).

Outre les 17 pays de concentration que compte la coopération suisse au développement, celle-ci continuera à insister sur les aspects concrets de sa politique étrangère sous la forme de programme spéciaux. Ces derniers ont pour principal objectif de permettre à la Suisse de soutenir les efforts des pays dans une situation de transition (passage d'une situation de guerre à un état de paix ou de stabilité, changement de système politique, etc.).

\section{Graphique 3: Répartition de la coopération bilatérale Engagements 1999-2002 (en millions de francs et en \% du total)}

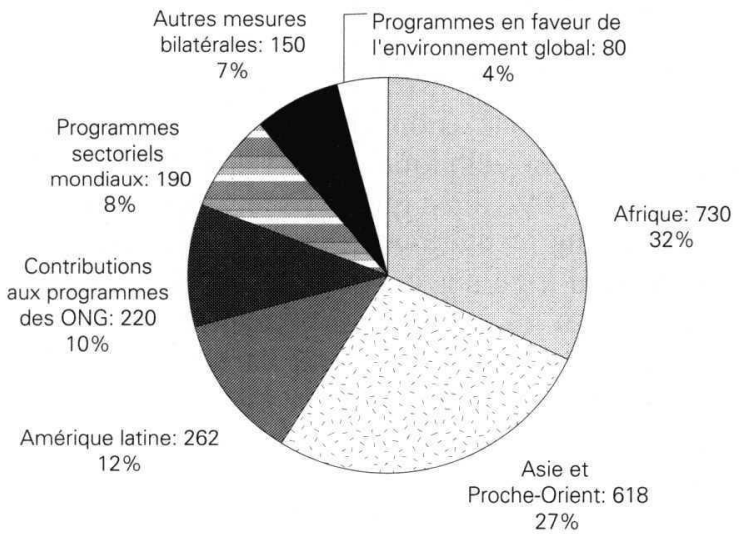

Source: à partir du « Message du Conseil fédéral concernant la continuation de la coopération technique et de l'aide financière» du 7 décembre 1998 (p. 126).

Au Rwanda, un programme spécial a été mis en œuvre pour améliorer le système judiciaire et promouvoir la réconciliation. Les territoires autonomes palestiniens bénéficient également d'un programme spécial. En 1994, la Confédération a démarré un programme d'aide avec l'Afrique du Sud pour surmonter les conséquences de l'apartheid. Ce programme spécial de 80 millions de francs, signé par 
le conseiller fédéral Flavio Cotti et le président Nelson Mandela, est limité à cinq ans car l'Afrique du Sud ne compte pas parmi les pays les pauvres auxquels s'adresse en priorité la coopération suisse. Ce programme a pour buts de soutenir le respect des droits humains, d'encourager la réforme agraire et de développer des initiatives dans le domaine de la formation.

La DDC deviendra plus sélective quant au choix de ses partenaires, en se montrant encore plus exigeante quant aux conditions de son aide. Ainsi, le concept de good governance deviendra un critère important de l'attribution des crédits d'aide (cf. le paragraphe plus haut sur la conditionnalité politique). Enfin, la coopération suisse continuera à collaborer étroitement avec des organisations non gouvernementales et avec l'économie privée, en Suisse comme à l'étranger.

\section{$\square$ Lignes directrices de la coopération multilatérale} au développement de la Suisse pour les années 1999 à 2002

L'apparition de problèmes globaux (migrations, pollution, drogue...) exacerbés par le processus de mondialisation pose une limite à la traditionnelle réglementation autonome des politiques nationales des Etats. Dans ce contexte d'interdépendance croissante du monde, la coopération multilatérale intervient comme une composante complémentaire de la coopération bilatérale, qui, du fait de la nature, de l'ampleur et de la portée des problèmes mondiaux, ne peut traiter seule de leur résolution.

L'adhésion aux organisations internationales donne par ailleurs à la Suisse la possibilité d'influencer et de surveiller la mise en œuvre des décisions des institutions concernées et de concourir à la résolution des principaux problèmes globaux. En outre, la contribution de la Confédération aux institutions et programmes multilatéraux permet à l'économie suisse de participer aux appels d'offres internationaux pour la livraison de biens et services.

Les priorités et recommandations énoncées dans le «Message concernant la continuation de la coopération technique et de l'aide financière en faveur des pays en développement» de 1994 ainsi que dans le «Message concernant la participation de la Suisse à l'augmentation du capital des banques de développement» de 1995 continueront à guider la coopération multilatérale pour les prochaines années. Toutefois, vu les restrictions budgétaires, la Confédération sera plus sélective qu'auparavant et plus exigeante dans la répartition des moyens financiers. Dans ce contexte et afin d'améliorer l'efficacité du système multilatéral actuel, caractérisé par de nombreux recoupements et doubles emplois, la Suisse privilégiera les aspects suivants: évaluer l'importance pour la coopération suisse de sa collaboration avec les diverses institutions multilatérales partenaires; renforcer la coordination et la coopération entre les institutions internationales les plus importantes; exploiter davantage leur synergie ainsi que les potentiels synergiques entre les projets et programmes bilatéraux et multilatéraux; et renforcer la présence de la Suisse au sein des équipes des institutions partenaires les plus importantes. La Suisse s'engagera également en faveur d'une répartition aussi juste que possible de la charge financière entre les donateurs des institutions internationales.

ASTM 1996, message sur la participation de la Suisse aux activités des banques régionales de développement (pp. 155-160). 
En outre, tout comme sur le plan bilatéral, le principe de concentration sera appliqué au niveau de la coopération multilatérale et se traduira par un choix encore plus sélectif des partenaires multilatéraux de la coopération suisse: il s'agira de répartir les ressources disponibles entre un nombre restreint d'institutions, afin d'obtenir, par une concentration des moyens, les meilleurs résultats possibles. Dans ce contexte, la Suisse continuera à coopérer avant tout avec les institutions internationales les plus importantes, soit la Banque mondiale, les trois plus importantes institutions du système onusien (PNUD, UNICEF et FNUAP), et les banques régionales de développement, dont plus particulièrement la Banque africaine de développement (voir plus loin le sous-chapitre 7.7). Les réformes en cours dans les banques régionales de développement seront un des thèmes centraux de la coopération multilatérale suisse pour la période 1999-2002, qui veillera particulièrement à ce qu'elles ne suivent pas des voies séparées et ne génèrent pas de doubles emplois.

Parallèlement, la coopération suisse continuera à coopérer avec les institutions multilatérales suivantes: le Groupe consultatif pour la recherche agricole internationale (CGIAR), le Fonds international de développement agricole (FIDA), le Fonds pour l'environnement mondial (FEM), l'Agence de la francophonie et le Comité d'aide au développement de l'OCDE.

\subsection{POLITIQUE DE LA DDC POUR LE DÉVELOPPEMENT SOCIAL}

En juin 2000 à Genève, sur invitation du Conseil fédéral, une session spéciale de l'Assemblée générale de l'ONU examinera la mise en pratique des engagements et des recommandations du Sommet mondial pour le développement social (Copenhague, mars 1995). En Suisse, le suivi du sommet a été réalisé par deux groupes de travail qui ont réuni des représentants des administrations fédérale et cantonales, des membres d'associations suisses et des universitaires: un groupe de travail, sous la direction du Département fédéral de l'intérieur, s'est intéressé aux actions à entreprendre en Suisse; l'autre groupe, sous la responsabilité de la DDC, s'est penché sur les questions relatives à la politique étrangère de la Confédération envers les pays du Sud et a publié son rapport en $1997^{10}$. Au cours de la période 1997-1998, la DDC a également procédé à une étude transversale de son approche de la lutte contre la pauvreté et de la promotion de l'empowerment ainsi qu'à un travail de clarification des concepts associés au développement social. Ces analyses ont abouti à l'élaboration de la Politique de la DDC pour le développement social ${ }^{11}$.

ASTM 1998, «Développement social» (p. 313).

Pour la DDC, une politique de développement social est une politique de société qui vise à éliminer la pauvreté dans toutes ses dimensions (économiques, matérielles, sociales, politiques et culturelles). En visant plus qu'une simple amélioration du revenu des indigents, elle doit s'intéresser aux dimensions politiques de la pauvreté par le biais de l'empowerment des populations défavorisées, qui peut déboucher sur une démocratisation véritable (pas seulement formelle), sur une

10. DDC, Le développement social dans les relations Nord-Sud, série Ecrits sur le développement, $\mathrm{n}^{\circ}$ 1, DDC, Berne, avril 1997.

11. DDC, document de travail 9-F/99, décembre 1999. 
bonne gestion des affaires publiques et sur une décentralisation qui serve les intérêts des pauvres. Ainsi, une politique de développement social, loin de se cantonner à des activités dans des secteurs dits sociaux (santé publique, éducation de base, sécurité alimentaire), doit toucher à la problématique de la structure du pouvoir.

La lutte contre des situations de pauvreté peut être effectuée par des méthodes directes ou indirectes. La lutte directe consiste à combattre, par l'aide humanitaire ou des programmes d'urgence, des situations de dénuement total. La lutte indirecte vise à améliorer dans son ensemble la situation de toutes les couches de la population et à renforcer des potentialités susceptibles d'être bénéfiques pour les pauvres. Parmi les mesures indirectes, jugées souvent plus utiles aux populations démunies que les interventions d'urgence de la lutte directe, figurent, par exemple, la démocratisation, la décentralisation, la promotion de la bonne gestion des affaires publiques, la lutte contre la corruption, la stabilisation des équilibres socio-économiques, etc. Dans sa politique pour le développement social, la DDC annonce aussi qu'elle étudiera la possibilité d'intégrer dans ses programmes des activités d'assistance sociale à long terme pour les populations en situation de détresse durable (réfugiés de longue date, enfants des rues), qui ne peuvent bénéficier de programmes de coopération au développement (dont les critères de sélection se basent sur l'existence de potentiels à moyen terme) ou de programmes d'aide humanitaire (cycles d'intervention courts). L'aboutissement des débats actuels sur de nouvelles sources de financement pour la solidarité internationale (taxe Tobin) permettrait à la communauté internationale de financer la mise en place de systèmes et d'instruments d'une assistance sociale à long terme.

Avec l'entrée en vigueur de la politique de la DDC pour le développement social le 15 janvier 1999, un groupe de travail interne «Développement social, lutte contre la pauvreté et empowerment» a été constitué dans le but de définir les modalités pratiques de la mise en œuvre de cette nouvelle politique.

\subsection{AIDE BILATÉRALE: COOPÉRATION TECHNIQUE ET AIDE FINANCIÈRE}

Entre 1997 et 1998, l'aide bilatérale a augmenté d'environ 7\%, passant de 841 millions de francs à 899 millions. Depuis 1994, la part de l'aide bilatérale dans l'APD de la Suisse diminue pour représenter moins de $70 \%$. Pour orienter la coopération bilatérale, les bases de travail de la DDC sont les documents d'orientation stratégique des sections géographiques, les papiers de réflexion stratégiques par thèmes ou secteurs, les programmes par pays et les programmes annuels.

Les programmes par pays (PPP) sont des programmes établis sur cinq ans par la DDC et le Seco. Pour chaque pays de concentration, ces programmes fournissent des informations sur la situation et les besoins de l'Etat en question. Ils précisent les objectifs généraux de la coopération suisse, les stratégies prévues et les ressources humaines et financières nécessaires pour atteindre ces objectifs. Les programmes annuels indiquent les étapes de la mise en œuvre de la coopération en précisant les articulations entre les différents niveaux d'intervention: micro (population), méso (institutions), macro (conditions-cadres), et permettent d'adapter les programmes par pays.

Les bureaux de coordination (BUCO) assurent la présence décentralisée de la DDC auprès de ces partenaires dans les pays dits de concentration. Proches des 
partenaires locaux et des réalités politico-administratives des pays d'intervention de l'aide suisse, ils jouent un rôle essentiel dans la conduite de la stratégie de la coopération suisse.

\section{$\square$ Répartition géographique de l'aide bilatérale}

Le tableau suivant montre que la coopération suisse se concentre avant tout sur les pays les plus pauvres.

Tableau 20: Répartition géographique de I'aide publique bilatérale par niveau de revenu des pays bénéficiaires (1998), en pour-cent*

\begin{tabular}{lccccr}
\hline & PMA & PFR ** $^{*}$ & PRITI** & PRITS** & Total \\
\hline Afrique & 78 & 4 & 7 & 11 & 100 \\
\hline Asie & 31 & 53 & 16 & - & 100 \\
\hline Amérique latine & 6 & 19 & 68 & 7 & 100 \\
\hline Europe de l'Est & - & 12 & 88 & - & 100 \\
\hline $\begin{array}{l}\text { Ensemble des } \\
\text { pays bénéficiaires }\end{array}$ & 43 & 27 & 25 & 5 & 100 \\
\hline
\end{tabular}

Source: à partir des données de Coopération internationale de la Suisse. Rapport annuel 1998, brochure "Coopération avec l'Europe de l'Est".

* Ce tableau concerne l'aide publique bilatérale spécifiquement affectée à des pays et exclut donc l'aide publique bilatérale affectée à des projets régionaux et à des projets non classifiables géographiquement.

** PFR, pays à faible revenu: pays dont le PNB par habitant était inférieur ou égal à 765 dollars en 1995; PRITI, pays à revenu intermédiaire de la tranche inférieure: pays dont le PNB par habitant se situait entre 766 et 3035 dollars en 1995; PRITS, pays à revenu intermédiaire de la tranche supérieure: pays dont le PNB par habitant se situait entre 3036 et 9385 dollars en 1995.

La répartition de l'aide publique bilatérale par continent varie par rapport aux chiffres des années précédentes. Les pays africains bénéficient, certes, toujours de la plus grande part des versements bilatéraux de la Suisse, mais celle-ci est en baisse ( $28 \%$ en 1998 contre un tiers en 1997). L'écart entre les versements bilatéraux destinés à l'Afrique et ceux attribués à l'Asie se réduit $(+7,8$ millions de francs en faveur du continent africain). Ainsi, les pays asiatiques ont reçu $26 \%$ de l'aide bilatérale suisse en 1998. L'Amérique latine et les pays du continent européen en développement ont bénéficié respectivement de $11 \%$ et $4 \%$ de l'APD bilatérale suisse.

En 1998, les dépenses totales imputables aux pays dits de concentration ${ }^{12}$ représentaient environ $47 \%$ de la totalité des sommes engagées dans la coopération bilatérale, en déduisant les contributions aux projets non classifiables géographiquement et aux projets régionaux. Ce pourcentage est proche de ceux que l'on peut noter en classant les pays de concentration par continent et en rapportant les versements bilatéraux versés à ces pays au total des versements bilatéraux versés pour leur continent respectif (exemple: en 1998, les pays de concentration situés sur le continent africain ont absorbé $42 \%$ de l'aide bilatérale suisse destinée à l'Afrique).

Les critères de concentration pour la coopération technique de la DDC ne s'appliquent pas à tous les autres volets de la coopération au développement. Ainsi, par exemple, les versements des bourses d'études, l'aide humanitaire, les mesures de politique économique et commerciale, les contributions aux ONG ne se dirigent pas prioritairement vers les pays dits de concentration. Le graphique 4

12. La politique de concentration de la DDC est détaillée plus haut, dans le paragraphe sur les axes prioritaires et thématiques de la coopération bilatérale. 
montre les principaux pays bénéficiaires de l'aide bilatérale suisse et met en évidence les différences dans les types d'aide reçus. Certains pays sont d'importants partenaires pour la coopération au développement (Népal, Bangladesh, Bolivie, Pakistan, Vietnam, etc.), alors que d'autres reçoivent beaucoup d'aide humanitaire (ex-Yougoslavie). Les mesures de politique économique et commerciale représentent une part importante de l'aide apportée à certains pays (Maurice, Chine, Nicaragua, Inde).

Graphique 4: Répartition de l'aide publique bilatérale suisse par instruments pour les 15 principaux pays bénéficiaires (1998), en millions de francs

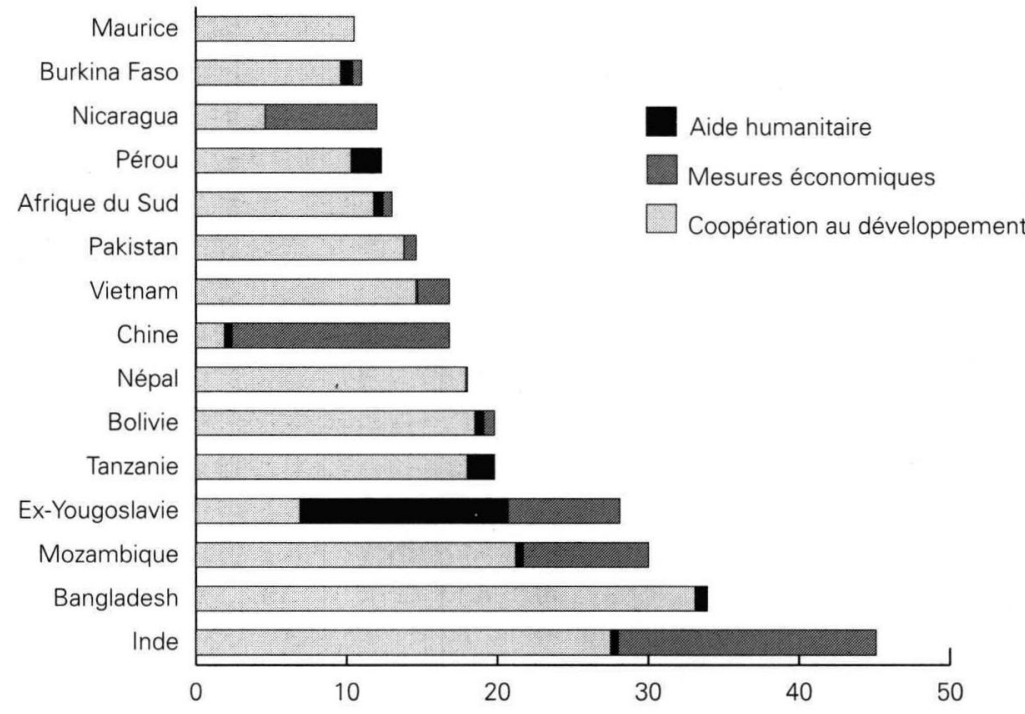

Source: à partir du tableau 3 de Coopération internationale de la Suisse. Rapport annuel 1998, brochure "Coopération au développement ".

\section{$\square$ Répartition sectorielle de l'aide bilatérale}

Par souci d'efficacité, l'essentiel de la coopération bilatérale se concentre sur un nombre limité de pays, mais aussi sur un nombre limité de secteurs. Le choix des actions de coopération bilatérale dans les pays en développement repose sur cinq grandes priorités: le renforcement de l'autonomie, le soutien aux couches de la population les plus pauvres, la maîtrise de l'environnement, l'amélioration des forces productives et la valorisation des ressources humaines. Les institutions des pays partenaires ont en règle générale l'initiative et la responsabilité des actions qui s'inscrivent dans leurs stratégies globales de développement. En 1998, les secteurs prioritaires de l'aide bilatérale versée par la DDC ont été l'infrastructure, l'eau et l'énergie (20\%), l'agriculture et l'élevage (15\%), l'éducation, l'information et la culture (13\%), la forêt et l'environnement $(12 \%)$ ainsi que la santé, la nutrition et la population (11\%).

\section{$\square$ Club du Sahel}

En septembre 1999, la Suisse, qui préside depuis deux ans le Club du Sahel en la personne d'Henri-Philippe Cart (vice-directeur de la DDC), a accueilli à Yverdon 
une grande conférence sur cette région de l'Afrique (qui s'étend du Tchad aux îles du Cap-Vert). Pendant trois jours, 12 pays donateurs et neuf pays africains se sont réunis, avec pour thèmes de réflexion les réformes des systèmes de l'aide internationale, les politiques de décentralisation dans les pays sahéliens, les pratiques de partenariat avec les organisations locales rurales et les dynamiques d'intégration régionale.

Le Club du Sahel a été créé en 1976 par les pays donateurs, les organisations internationales et les Etats sahéliens membres du Comité permanent inter-Etats de lutte contre la sécheresse au Sahel (CILSS). Le Club du Sahel, dont le secrétariat est installé à Paris au siège de l'OCDE, est un forum de dialogue, de concertation et de partenariat entre les pays de l'OCDE et les pays sahéliens, permettant une réflexion approfondie sur les principaux enjeux de l'avenir du Sahel, un partage des connaissances et des expériences et une remise en question des idées reçues. Le Club du Sahel a donc pour mission de favoriser une compréhension commune du Sahel entre les partenaires au développement, d'anticiper les changements à court et à long terme, et de promouvoir par conséquent l'efficacité de la coopération pour le développement des pays sahéliens. Orientés il y a vingt ans vers la lutte contre les pénuries alimentaires, les programmes de travail du Club du Sahel sont aujourd'hui diversifiés pour répondre aux évolutions de la démographie, de l'environnement, de la mondialisation économique et de la démocratie.

A l'automne 1997, les membres du Club du Sahel ont adopté le «Mémorandum de Banjul», un second contrat de génération entre les pays sahéliens et leurs partenaires internationaux de l'OCDE, faisant suite au contrat de génération qui avait présidé à la création du club vingt plus tôt. Ce nouveau contrat insiste sur la nécessité d'accroître la participation des acteurs de la société civile à la définition et à la mise en œuvre des stratégies et des actions de développement. La coopération suisse, qui alloue chaque année environ 70 millions de francs d'aide au Sahel, s'inscrit dans cette perspective. Ses domaines d'intervention sont le développement rural, l'artisanat, l'éducation, la santé et la gestion publique. Différents programmes de la DDC soutiennent au Sahel les groupements de base ruraux, favorisent les échanges entre le secteur public et privé, et accompagnent les gouvernements dans leurs efforts de redéfinition du rôle de l'Etat. En tant que présidente du Club du Sahel depuis 1997, la DDC a régulièrement porté le débat sur la réforme des instruments de l'aide au service des projets des Sahéliens.

\subsection{AIDE BILATÉRALE: MESURES DE POLITIQUE ÉCONOMIQUE ET COMMERCIALE}

Le cinquième crédit de programme pour la continuation et la réorientation des mesures de politique économique et commerciale est entré en vigueur au début de l'année $1997^{13}$. Ces mesures de coopération sont gérées par le Secrétariat d'Etat à l'économie (Seco). Celui-ci, dépendant du Département fédéral de l'économie, a ainsi géré en 1998 12\% de l'APD (8\% en 1997). Les objectifs du programme de coopération économique au développement géré par le Seco sont les suivants:

13. «Message concernant la continuation du financement et la réorientation des mesures de politique économique et commerciale au titre de la coopération au développement» du 29 mai 1996 (message n 96.044). 
a aider les pays en développement à établir les conditions-cadres propices à la croissance et à l'investissement, notamment par les aides à la balance des paiements et par les opérations de désendettement;

๖ mobiliser les ressources du secteur privé afin d'accroître les flux de financement et le transfert de technologies;

」 mieux intégrer les pays en développement dans les échanges internationaux.

Le tableau suivant montre la répartition des versements du Seco par type d'instruments de coopération, de 1994 à 1998.

Tableau 21: Mesures de politique économique et commerciale (1994-1998)

\begin{tabular}{lrrrrrr}
\hline Types & \multicolumn{7}{c}{ Versements en millions de francs } & En \% du total \\
de mesures & $\mathbf{1 9 9 4}$ & $\mathbf{1 9 9 5}$ & $\mathbf{1 9 9 6}$ & $\mathbf{1 9 9 7}$ & $\mathbf{1 9 9 8}$ & $\mathbf{1 9 9 4 - 1 9 9 8}$ \\
\hline $\begin{array}{l}\text { Aide à la balance } \\
\text { balance des paiements }\end{array}$ & 29.2 & 13.0 & 29.5 & 40.2 & 10.5 & 17.3 \\
\hline $\begin{array}{l}\text { Mesures } \\
\text { de désendettement }\end{array}$ & 41.4 & 68.4 & 38.1 & 21.2 & 40.4 & 29.7 \\
\hline $\begin{array}{l}\text { Promotion commerciale } \\
\text { Produits de base }\end{array}$ & 5.1 & 7.5 & 13.5 & 4.8 & 10.2 & 5.8 \\
\hline $\begin{array}{l}\text { Promotion } \\
\text { des investissements }\end{array}$ & 23.5 & 7.3 & 3.3 & 3.6 & 3.4 & 3.0 \\
\hline $\begin{array}{l}\text { Financements mixtes } \\
\text { Etudes et administration }\end{array}$ & 75.4 & 26.9 & 27.7 & 9.5 & 22.7 & 19.0 \\
\hline $\begin{array}{l}\text { Total des } \\
\text { mesures économiques }\end{array}$ & 180.2 & 135.9 & 135.7 & 103.2 & 151.7 & 23.0 \\
\hline
\end{tabular}

Source: données du Seco.

\section{$\square$ Aide à la balance des paiements}

Cette aide permet de financer des importations de première nécessité en provenance de la Suisse et, plus généralement, d'assister financièrement les gouvernements bénéficiaires engagés sur la voie de réformes (ajustement structurel par exemple). Cette assistance, de même que les mesures de désendettement, est accordée de manière sélective aux pays démontrant un solide engagement dans la mise en œuvre de réformes économiques.

En 1998, un nouvel accord a été conclu avec le Mozambique pour un montant total de 18 millions de francs (cinquième aide à la balance des paiements avec ce pays). Un accord bilatéral portant sur une aide à la balance des paiements de 5 millions de francs en faveur de l'Albanie a été signé le 12 novembre 1999. Cette mesure d'urgence vise à couvrir les besoins de financement additionnels causés par l'afflux massif de réfugiés du Kosovo au début de l'année 1999. L'impact additionnel de cette crise sur la balance des paiements de l'Albanie avait été estimé par le FMI et la Banque mondiale à 133 millions de dollars. Aucun autre nouvel accord d'aide à la balance des paiements n'a été conclu en 1999, mais les pays suivants ont bénéficié de versements en 1999 en vertu d'accords en vigueur: Burkina Faso, Cameroun, Mozambique, Ouganda, Tanzanie, Sénégal, Bolivie, Nicaragua et Vietnam.

\section{$\square$ Mesures de désendettement}

Le Programme de désendettement a été instauré en 1991 lors de l'adoption du crédit-cadre du $700^{\mathrm{e}}$ anniversaire de la Confédération. 400 millions de francs ont 
été affectés au soutien des mesures de désendettement. Ce programme, prévu à l'origine pour une durée de cinq à sept ans, a été prolongé et reste encore en vigueur. Le Seco est chargé de la mise en œuvre du programme de désendettement, en collaboration avec la DDC et les ONG suisses. La Suisse participe activement à la mise en œuvre de l'initiative de désendettement global de la Banque mondiale et du FMI en faveur des pays pauvres très endettés (PPTE). Quatre pays ont pu bénéficier jusqu'à présent d'une réduction de dettes dans le cadre de cette initiative (Bolivie, Guyane, Mozambique et Ouganda), et la Suisse soutient l'élargissement de l'initiative à d'autres pays. Avec d'autres pays européens (Autriche, Danemark, Grande-Bretagne et Suède), elle accompagne la mise en œuvre de l'initiative PPTE avec un programme d'assistance technique. En 1999, la Suisse a soutenu (à hauteur de 10 millions de francs) une action de désendettement commercial en faveur de la Tanzanie (action coordonnée par l'AID). Elle e a aussi pris part à des actions de réduction des dettes multilatérales du Burkina Faso et de la Tanzanie (respectivement pour 6 et 7 millions de francs). Elle a contribué à l'allègement du service de la dette multilatérale du Honduras pour un montant de 15 millions de francs (afin d'aider ce pays durement touché par l'ouragan «Mitch»).

ASTM 2000, le chapitre 1 de la partie «Revue» détaille la politique de la Suisse au sein des institutions de Bretton Woods, les mesures de désendettement prises sur le plan multilatéral et les mesures suisses dans ce domaine.

\section{$\square$ Promotion des investissements dans les pays en développement ou en transition}

Le crédit de programme proposait une série de nouveaux instruments de coopération destinés à stimuler les investissements vers les pays en développement ou vers les pays en transition.

La SDFC (Swiss Development Finance Corporation) a été créée au printemps 1999 avec pour but de favoriser les flux d'investissements privés à long terme dans les pays en développement et en transition. Le capital (55 millions de francs lors de la création, devant atteindre à terme 100 millions) est détenu à $51 \%$ par des investisseurs privés ${ }^{14}$ et à $49 \%$ par le Seco. La SDFC entend aider les PME suisses à obtenir les crédits nécessaires pour investir dans ou créer des jointventures dans ces régions.

Dans sa prise de position, la Déclaration de Berne estime que la SDFC ne se préoccupe, pour l'instant, que des intérêts de l'économie privée et néglige les aspects relatifs à la politique de développement ${ }^{15}$. Soutenir les PME avec de l'argent de la coopération au développement n'a de sens, selon la Déclaration de Berne, que si ce soutien répond aux besoins des pays les plus pauvres. Les expériences faites dans les institutions comparables de la Banque mondiale (Société financière internationale - SFI; Agence multilatérale de garantie des investissements - AMGI) montrent que la promotion du secteur privé doit être accompagnée par des directives sociales et écologiques contraignantes pour parvenir à un impact positif en matière de développement, directives qui ne sont pas encore établies pour la SDFC. Les flux de capitaux privés se concentrent sur quelques pays émergents alors que les 48 PMA ne reçoivent que $1 \%$ de tous les investis-

14. Il s'agit de l'UBS, du Zurich Financial Services Group, ainsi que d'un groupe de banques cantonales (AG, BL, GE, LU, SG, TG, VD, ZH). Le conseil d'administration de la SDFC est présidé par le directeur général de l'UBS; la viceprésidence est un représentant du Seco. La société spécialisée Swiss Emerging Markets Partners AG (SEMP, Zurich) assure la direction opérationnelle de la société. Adresse de la SDFC: Talacker 41, case postale, 8039 Zurich; tél. 061/226.52.60. Site Internet: www.swiss-dfc.ch.

15. On peut consulter la prise de position de la Déclaration de Berne sur le site Internet www.evb.ch/db. 
sements directs étrangers. Au lieu de renverser la tendance, la SDFC se concentrera aussi sur les pays à forte croissance économique et sur des secteurs déjà en forte progression (énergie et télécommunications par exemple). L'histoire de la création de la SDFC - prévue en 1995 déjà - donne aussi l'impression que l'économie privée ne fait preuve pour l'instant que d'un intérêt limité pour cet instrument.

La fondation à but non lucratif SOFI (Swiss Organization for Facilitating Investments) a été créée par l'OFAEE (actuellement Seco) en $1997^{16}$. Les buts de la SOFI sont de faciliter la création de liens économiques entre les PME suisses et des entreprises des pays en développement ou en transition, ainsi que d'encourager l'augmentation des flux d'investissements, de know-how industriels et de technologies de la Suisse vers ces pays. Les services de SOFI comprennent les éléments suivants:

๖ promotion des investissements: organisation de conférences, élaboration et diffusion d'informations sur les opportunités d'investissements dans les pays partenaires;

口 services d'information: sur la situation politique et économique, les conditions-cadres, le marché du travail, les systèmes douaniers et fiscaux dans les pays partenaires, et sur les sources de financement disponibles en Suisse;

- services de recherche et de sélection de partenaires d'affaires: mise en relation d'affaires, assistance dans la première phase de préparation de projets et assistance opérationnelle dans la réalisation;

- consulting: sur les politiques d'investissement, la formulation de projets, les études de faisabilité, la formation...

Deux fonds de capital-risque ont été créés en 1997 par la Confédération, en Inde (Swiss Technology Venture Capital Fund ${ }^{17}$ ) et en Chine (Sino-Swiss Partnership Fund $\left.^{18}\right)$. Un autre fonds de capital-risque existe pour les PME travaillant en Afrique du Sud ${ }^{19}$. Ces fonds permettent à des entreprises indiennes, chinoises ou sud-africaines de diversifier ou d'étendre leurs activités en établissant des liens avec des entreprises suisses (soutien de joint-ventures). La Suisse fait partie des 10 pays plus grands investisseurs en Inde ( $4^{\mathrm{e}}$ plus grand investisseur européen derrière le Royaume-Uni, les Pays-Bas et l'Allemagne). Ele se situe parmi les principaux pays investisseurs en Chine et est au $6^{c}$ rang pour l'Afrique du Sud. Les investissements directs étrangers sont surtout le fait des grandes entreprises suisses (qui n'ont pas besoin de la coopération suisse au développement pour se faire connaître), mais les nouveaux instruments du Seco cherchent à appuyer les efforts des PME suisses dans ces pays. Le fonds en Inde permet par exemple d'appuyer des projets dans une fourchette de 350'000 à 1,7 million de francs, ce qui est particulièrement intéressant pour les PME.

En collaboration avec d'autres institutions financières internationales, le Seco a participé au capital de la Tuninvest International Ltd., active en Tunisie pour mettre des capitaux à la disposition d'entreprises tunisiennes. La ligne de crédit

16. Adresse: Stauffacherstr. 45,8004 Zurich, tél. 01/249.30.50. Site Internet: www.sofi.ch.

17. Site Internet: www.sofi.ch/financial/swisstec_venture_capital_fund.htm.

18. Site Internet: www.seco-admin.ch/entwicklung/f_invest/edpeco/partner1.htm.

19. Msele NedVentures LTD. Voir le site Internet www.sofi.ch/financial/msele_nedventures_ltd.htm pour plus d'informations. 
ouverte dans le cadre du Partenariat tuniso-suisse a pour but de soutenir les projets d'investissement de PME tuniso-suisse installées en Tunisie (joint-ventures). Le fonds permet par exemple de financer des crédits (de 100'000 à 2 millions de francs au maximum) servant à financer des importations de biens d'équipement et/ou de services d'origine suisse utilisés dans le projet d'investissement, ou des fonds de roulement liés au projet d'investissement.

Le Seco a également participé au Terra Capital Investor Ltd. ${ }^{20}$, qui assure la promotion d'investissements dans le domaine de la biodiversité. Ce fonds, créé par la Société financière internationale en collaboration avec le Fonds pour l'environnement mondial (FEM), vise à prendre des participations en capital dans des PME d'Amérique latine quand leurs projets d'investissement visent à sauvegarder la diversité biologique (les études de faisabilités, évaluations techniques et l'assistance technique peut être financée par le FEM).

Le Seco participe aussi au financement de plusieurs centres de technologies propres (national cleaner production centers), qui ont pour but de promouvoir le transfert de technologies respectueuses de l'environnement. Des centres de ce type ont été créés par exemple en Colombie ${ }^{21}$, au Brésil, au Guatemala, au Maroc et au Vietnam (préparation d'un centre au Pérou).

L'accord portant sur la création du centre de São Paulo a été signé en juillet 1999 lors de la visite du conseiller fédéral Pascal Couchepin au Brésil; les partenaires en sont le Seco, la Fédération des industries de l'Etat de São Paulo et l'Institut de formation de la fédération nationale des industries. Le centre conseille les entreprises locales sur la gestion de l'environnement, sur l'efficacité énergétique et le choix d'appareils et de technologies ménageant l'environnement, et sur les services disponibles en Suisse. L'objectif est de mieux respecter la législation sur la protection de l'environnement et d'aider les entreprises brésiliennes à observer les normes écologiques nécessaires à l'accès aux marchés du Nord. Les entreprises suisses disposant d'un savoir-faire dans le domaine des technologies respectueuses de l'environnement, le Seco souhaite, par ce type d'instrument de coopération, favoriser le transfert de ces technologies vers les pays en développement. Chaque cleaner production center (CPC) dispose d'un point de référence en Suisse (école polytechnique ou université par exemple), l'institution suisse mettant le CPC en relation avec les détenteurs de technologies (industries suisses, bureaux d'ingénieurs-conseils) et le soutenant par une assistance technique.

Le Fonds pour l'étude de projets de financement permet, comme son nom l'indique, de financer la préparation d'études pour des nouveaux projets d'investissement $^{22}$. Le financement du Seco peut couvrir au maximum $50 \%$ des coûts, pour un montant maximal d'un million de francs. Le crédit est remboursable si l'étude débouche effectivement sur un nouvel investissement et transformable en don si aucun investissement ne s'en est suivi.

\section{$\square$ Promotion du commerce avec les pays en développement ou en transition}

Les mesures de soutien dans le domaine des produits de base ont perdu de leur importance suite à la suppression des mécanismes de soutien des prix dans tous

20. Site Internet: www.bancoaxial.com.

21. «La Suisse ouvre en Colombie le Centre de promotion des techniques de production respectueuses de l'environnement», Communiqué de presse de l'OFAEE, 18.3.98; «La Suisse lance un Cleaner Production Center au Brésil», Communiqué de presse du Seco, 7.7.99.

22. Pour plus de renseignements, voir le site Internet www.seco-admin.ch/entwicklung/f_invest/edpeco/study.htm. 
les accords internationaux sur les produits de base. La coopération de la Suisse dans ce domaine porte essentiellement sur le financement de projets d'exploitation durable des bois tropicaux, dans le cadre de l'Organisation internationale des bois tropicaux. La Suisse soutient des projets dans certains pays d'Afrique et d'Amérique latine, ainsi que dans les régions de l'Amazonie, du bassin du Congo et du Sarawak (Bornéo).

En mai 1999, le Seco a renouvelé son soutien à l'OSEC pour poursuivre la promotion des importations en provenance des pays en développement ou en transition (nouveau mandat de 26 millions de francs pour quatre ans). Le nouveau programme se concentre sur un nombre restreint de pays partenaires et de secteurs économiques, et réunit les programmes autrefois séparés au bénéfice des pays en transition et des PVD en une seule entité, le SIPPO (Swiss Import Promotion Programme, intégré à l'OSEC ${ }^{23}$ ). Il ne s'agit pas de subventionner des exportations, mais de faciliter les démarches pour les exportateurs potentiels (en particulier les PME) dans les pays en développement et en transition. Ce Programme suisse de promotion des importations diffuse des informations sur le marché suisse (renseignements sur le marché pour un produit ou un secteur spécifique, droits de douane, prescriptions en matière d'emballage et d'étiquetage), offre des services de mise en relation d'affaires (recherche ciblée de partenaires potentiels) participe à l'organisation de manifestations (forums de contacts, participation à des foires spécialisées, formation). Le Seco va aussi établir des programmes spécifiques par pays ou par régions (projet pilote en Bolivie).

Rappelons que la part des pays en développement dans les importations de la Suisse est très faible. Ces pays représentaient $8,7 \%$ des importations totales de la Suisse en 1997, et les importations en provenance des pays de l'Europe centrale et orientale ne représentaient que $2 \%$ des importations suisses. La promotion des exportations des pays en développement vers les pays industrialisés est aussi soutenue par la Suisse par le biais de la CNUCED (notamment par les trade points et la formation) et du Centre du commerce international (CCI, organisation conjointe à l'OMC et la CNUCED), et du financement d'initiatives du commerce équitable (par exemple, financement du lancement du label Max Havelaar).

\section{$\square$ Financements mixtes}

Les financements mixtes combinent un don de la Confédération et un prêt (aux conditions du marché) consenti par un consortium de banques suisses. Ces crédits permettent aux pays partenaires d'acheter des biens d'équipement et des services en Suisse (pour $50 \%$ au minimum du crédit) ou dans d'autres pays.

Cet instrument de coopération a perdu de son importance depuis le milieu des années 90 suite à l'adoption en 1992 des règles d'Helsinki par les pays membres de l'OCDE. Les entreprises suisses éprouvent de la difficulté à identifier des projets conformes aux nouvelles règles établies par l'OCDE. Celles-ci restreignent le recours aux financements mixtes à des projets commercialement non viables. Avec 10 millions de francs en 1997, les déboursements liés aux financements mixtes ont atteint leur niveau le plus bas, pour se stabiliser à 25 millions de francs

23. Adresse du SIPPO: 4, rue de l'Avant-Poste, 1001 Lausanne; tél. 021/320.32.31. Voir aussi «Hilfe zum Handel als Entwicklungsbeitrag», Neue Zürcher Zeitung, 11.5.99. 
environ en 1998 et 1999. La grande partie des déboursements durant ces années ont été occasionnés pour le financement de projets d'infrastructure dans le cadre des accords de financement mixte avec la Chine, le Vietnam et l'Egypte ( $4^{\mathrm{e}}$ crédit mixte d'un montant de 80 millions de francs en vigueur depuis le début de 1999).

\subsection{AIDE MULTILATÉRALE}

Depuis les années 90, l'aide multilatérale est en constante augmentation. Elle a atteint 388 millions de francs en 1998 et sa part dans l'APD suisse est d'environ $30 \%$. Il faut toutefois noter que l'aide comptabilisée comme étant multilatérale ne recouvre pas toute l'aide transitant par les organisations internationales. En effet, l'aide est multilatérale lorsque la contribution d'un pays à une organisation internationale se fond avec les contributions d'autres pays et que l'institution en question décide seule de l'affection de ces ressources. L'aide associée, les cofinancements $^{24}$ et les contributions au CICR représentent des versements multi-bilatéraux et sont comptabilisés dans l'aide bilatérale. L'aide associée et les cofinancements se sont élevés à près de 84 millions de francs en 1998. Avec les versements au CICR (environ 82 millions de francs), c'est au total plus de 550 millions de francs qui transitent par les organisations internationales (soit $43 \%$ de l'APD totale).

Près de 50\% de l'aide multilatérale aux pays du Sud a été versée en 1998 aux banques de développement (le groupe de la Banque mondiale et les trois banques régionales concernées) et à leurs fonds spéciaux. L'Association internationale de développement (AID) a reçu la plus grande part de l'aide multilatérale affectée aux institutions financières de développement (106,6 millions de francs, soit environ $56 \%$ ).

La Suisse n'est pas membre des Nations Unies, mais elle soutient les activités des principaux programmes, fonds et organes subsidiaires de l'organisation. Ainsi, les contributions au PNUD (56 millions de francs), au PAM (18,1 millions), à l'UNICEF (17 millions), au HCR (13 millions) et au FNUAP (10 millions) ont représenté presque 30\% de l'APD multilatérale suisse en 1998.

D'autres institutions multilatérales, telles que le Groupe consultatif pour la recherche agricole internationale (CGIAR) ou le Fonds international de développement agricole (FIDA), bénéficient également de l'aide multilatérale suisse.

\section{$\square$ Banques régionales de développement}

(africaine, asiatique et interaméricaine) ${ }^{25}$

Les banques régionales de développement sont des institutions financières multilatérales dont la mission première consiste à soutenir des projets et des programmes pour réduire la pauvreté et promouvoir le développement durable. Pour ce faire, ces institutions remplissent les fonctions principales suivantes:

- elles représentent dans beaucoup de pays du Sud une des plus importantes sources de capitaux par le biais des prêts qu'elles octroient aux pays les plus

24. «Aide associée»: financement de projets administrés par une organisation internationale, mais le pays bailleur de fonds choisit les projets. «Cofinancements»: actions financées simultanément par plusieurs pays donateurs.

25. Pour la Banque mondiale, se référer au chapitre 1 de la «Revue», et pour la BERD, au chapitre 9. 
avancés de leur région à des conditions proches de celles du marché et par le biais des fonds de développement réservés aux pays les plus pauvres. A ces prêts peuvent s'ajouter des contributions spécifiques des bailleurs de fonds publics ou privés pour des programmes ou projets individuels;

- elles soutiennent, avec les institutions de Bretton Woods, des programmes d'ajustement macro-économique et de réformes sectorielles;

- elles financent, par le biais des guichets concessionnels, des projets socioéconomiques à faible rentabilité financière (gestion de l'eau, santé, éducation, etc.);

- elles offrent des prestations importantes sous forme de conseils techniques et de mesures spécifiques dans le but de renforcer les capacités locales d'administration et de gestion.

Les ressources des banques régionales de développement sont composées du capital-actions de base, des emprunts effectués sur le marché international des capitaux, des contributions des pays donateurs aux fonds de développement, ainsi que des revenus de placements à court terme. Ces institutions sont dirigées par un conseil des gouverneurs (dans lequel chaque pays membre siège avec un gouverneur et un suppléant) et un conseil d'administration (avec des administrateurs représentant un groupe de pays), et elles sont gérées par une direction.

La Suisse est membre de la Banque asiatique de développement depuis 1967 (57 pays membres dont 16 pays non régionaux), de la Banque interaméricaine de développement depuis 1976 (46 pays membres dont 18 pays non régionaux) et de la Banque africaine de développement depuis 1982 (77 pays membres dont 24 pays non régionaux). Pour la Suisse, sa participation à ces trois banques (mise à disposition de ressources financières et de garanties) élargit sa solidarité à des pays en développement avec lesquels elle n'a pas de programme de coopération bilatérale. Le Parlement a approuvé en 1995 un crédit de programme concernant la participation de la Suisse à l'augmentation du capital des trois banques régionales ainsi que de la Société interaméricaine d'investissement (SII) et de l'AMGI. Ce crédit-cadre de 800 millions de francs (dont 755 millions constituent un capital de garantie) est la base légale des engagements de la Suisse. En 1998, les contributions de la Suisse aux banques régionales de développement et à leurs fonds spéciaux ont été d'environ 80 millions de francs.

En tant que membre, la Suisse est représentée directement ou indirectement au conseil d'administration de ces institutions en fonction du montant de sa participation. Ainsi, elle participe à:

- l'élaboration des politiques sectorielles en faisant entendre celles qu'elle considère comme prioritaires telles que la lutte contre la corruption, la promotion du secteur privé, l'environnement, etc.;

- la discussion et l'approbation des programmes et projets, en concentrant ses prises de position sur des programmes et projets dont, premièrement, la mise en œuvre touche des secteurs prioritaires pour elle et qui, en second lieu, sont menés dans les pays privilégiés de sa politique de développement;

- la prise de position sur les questions institutionnelles (politique de gestion, budget, évaluations des activités, politique d'information, etc.).

Selon le «Message du Conseil fédéral concernant la continuation de la coopération technique et de l'aide financière en faveur des pays en développement» du 
7 décembre 1998, la Suisse axera, pour les quatre prochaines années, ses efforts sur la Banque africaine de développement, en la soutenant dans l'amélioration de la qualité de ses opérations et en favorisant un meilleur équilibre entre les intérêts régionaux et non régionaux au sein de la banque. En outre, pour améliorer la collaboration au sein des organes de direction des trois institutions financières régionales, la DDC et le Seco établiront (comme pour les institutions de Bretton Woods) une plate-forme commune en vue d'une coopération entre les trois banques.

\section{Banque africaine de développement}

Après avoir traversé une crise aiguë de gestion au début des années 90, la Banque africaine de développement (BAD) s'est engagée depuis 1995 dans un processus de réformes institutionnelles et financières. De l'avis de la Confédération, le groupe connaît désormais un retour à la normale de ses activités opérationnelles. Pour améliorer la qualité de ses opérations, la banque a élaboré en 1998 et en 1999 un certain nombre de politiques et de directives. Celles-ci portent notamment sur le développement agricole et rural, la santé, la bonne gouvernance ainsi que les ressources en eau. Après deux années de négociations laborieuses, l'adoption en 1998 d'un accord sur l'augmentation de $35 \%$ du capital de la banque (passant de 21 milliards de dollars à 29 milliards de dollars) a contribué à consolider son assise financière sur les marchés internationaux de capitaux. Suite à cet accord entré en vigueur en septembre 1999, la part des pays non régionaux dans le capital total de la banque est passée d'un tiers à $40 \%$, et celle des pays régionaux de deux tiers à $60 \%$.

La huitième reconstitution générale du Fonds africain pour le développement (FAD-VIII) s'est effectuée en un temps record, témoignant de la confiance renouvelée des actionnaires dans la banque. Pour le financement d'opérations concessionnelles pour la période 1999-2001, les ressources du FAD ont été reconstituées à la hauteur de 3,4 milliards de dollars en 1999. Le Conseil fédéral a approuvé en septembre 1999 la participation de la Suisse au FAD-VIII, avec une contribution non remboursable de 138 millions de francs. Pour le Conseil fédéral, la Suisse manifeste ainsi sa volonté d'assumer sa part dans un effort commun des pays donateurs en faveur des pays pauvres de l'Afrique. Le seul pays membre régional à avoir souscrit au FAD-VIII est l'Afrique du Sud. Les pays régionaux ont été invités à suivre cet exemple à l'occasion de la tenue conjointe, en mai 1999 au Caire, de la 35 ${ }^{\circ}$ Assemblée annuelle de la BAD et de la $26^{\circ}$ Assemblée annuelle du FAD. L'allocation des ressources du FAD-VIII aux pays bénéficiaires dépendra de la performance et de la bonne gouvernance de chaque pays. L'orientation stratégique du fonds se concentrera sur la bonne gestion des affaires publiques, sur la mise en place d'un environnement favorable à l'initiative privée, sur l'intégration systématique de la promotion de l'égalité femmes-homme dans les projets, sur la gestion durable des ressources naturelles et, enfin, sur le renforcement de la coopération et l'intégration régionales.

Enfin, le conseil d'administration de la banque a approuvé la création, à Abidjan, d'un Institut conjoint pour l'Afrique (ICA) en coopération avec le FMI et la Banque mondiale. Cet institut sera chargé de la formation des fonctionnaires des pays membres régionaux dans les principaux domaines d'intérêt de la banque et de l'Afrique. 


\section{Banque asiatique de développement}

Les montants des prêts accordés par la Banque asiatique de développement (BAsD) en 1998 ont retrouvé leur niveau de 1996. La BAsD a approuvé un volume de prêts s'élevant à environ 6 milliards de dollars comprenant 4,9 milliards de dollars de prêts sur capital ordinaire et 987 millions de dollars de prêts concessionnels. Le niveau record des prêts accordés par la BAsD en 1997 (9,4 milliards de dollars) s'expliquait par le financement d'opérations de sauvetage d'urgence pour contrecarrer les effets néfastes de la crise asiatique dans certains pays. Les principaux bénéficiaires ont été la Corée du Sud (avec un prêt de 4 milliards de dollars, soit le plus important prêt consenti par la banque depuis sa création), l'Indonésie (1,1 milliard de dollars) et la Thaïlande (500 millions de dollars). Ces prêts ont prioritairement financé la réforme des secteurs financier et bancaire des pays en crise.

En 1998, les ressources utilisées pour la réforme des marchés financiers ont encore constitué une grande part des prêts accordés (28\%). Un quart de ces derniers ont été destinés à des opérations dans le secteur des transports et des communications. Les investissements dans le secteur des infrastructures sociales représentent seulement 11\% des crédits (en forte baisse depuis 1996 alors que les pays membres de la banque s'étaient fixé l'objectif de 50\% des crédits dans ce secteur). Pour certains pays membres de la BAsD, la mobilisation massive de ressources pour des interventions d'urgence pose la question de la mission première de la banque, qui devrait être la lutte contre la pauvreté et la promotion du développement à long terme. Les principaux bénéficiaires des prêts sur capital ordinaire ont été l'Indonésie et la Chine, absorbant en 1998 plus de $60 \%$ des ressources financières ordinaires de la BAsD. Le Fonds asiatique pour le développement (FAsD) a fourni des prêts concessionnels pour 987 millions de dollars (contre 1,6 milliard l'année précédente). Près de la moitié des ressources concessionnelles ont été versées à trois pays: le Bangladesh, le Vietnam et le Népal.

\section{Banque interaméricaine de développement}

Pour la cinquième année consécutive, la Banque interaméricaine de développement (BID) est le principal bailleur de fonds multilatéral de l'Amérique latine et des Caraïbes. Le montant total des prêts accordés par la BID (prêts sur le capital ordinaire et prêts concessionnels) a atteint en 1998 un niveau record, environ 10 milliards de dollars contre 6 milliards de dollars l'année précédente. Cette augmentation de $67 \%$ s'explique en grande partie par l'introduction de nouveaux prêts financiers d'urgence. En effet, pour atténuer les effets des crises financières asiatique et russe sur l'Amérique latine et pour assurer aux catégories sociales les plus défavorisées une protection face aux chocs causés par l'instabilité financière mondiale, l'assemblée des gouverneurs de la banque a approuvé la création d'un programme de prêts d'urgence se situant en marge de son programme ordinaire de prêts et bénéficiant d'une dotation pouvant atteindre 9 milliards de dollars. Les prêts d'urgence, avec une échéance de cinq ans et un délai de grâce de trois ans, sont supportés par le capital ordinaire de la banque et décaissés dans des délais très courts. En 1998, la BID a approuvé deux opérations financières d'urgence. La première, qui représente un prêt de 2,5 milliards de dollars (le prêt le plus important consenti par la banque depuis sa création), finance un programme en faveur de l'ajustement sectoriel et des mesures de sauvegarde du système bancaire en Argentine. La seconde opération affecte 350 millions de dollars à un programme de réforme du secteur de l'électricité en Colombie. L'augmentation de 
l'aide consentie par la BID s'explique également par sa contribution (800 millions de dollars) aux dispositifs de secours et aux programmes de redressement mis en place suite aux ravages causés par les cyclones «George» et «Mitch» en Amérique centrale et dans les Caraïbes.

Même en excluant les prêts d'urgence, le montant des prêts de la BID en 1998 était un des plus importants de son histoire. La plus grande partie des prêts a été destinée à la lutte contre la pauvreté; $46 \%$ des prêts ont été consacrés à des programmes sociaux tels que l'éducation et la santé. La banque a également largement dépassé ses objectifs dans la promotion du secteur privé: elle a accru ses autorisations de prêts et de garanties en faveur de ce secteur, qui ont atteint un niveau record d'environ 566 millions de dollars (contre 320 millions de dollars en 1997), dont 215 millions de prêts aux très petites entreprises (TPE).

La 40 Assemblée annuelle de la BID s'est tenue à Paris en mars 1999, en même temps que la $14^{\mathrm{c}}$ Assemblé annuelle de la Société interaméricaine d'investissement. La SII est une société autonome d'investissement affiliée à la BID, dont l'objectif est la promotion du développement des PME dans la région. Cette $14^{\circ}$ Assemblée a approuvé une augmentation de capital de 500 millions de dollars, portant celui-ci à 700 millions de dollars.

Dès l'an 2000, la question des ressources du Fonds concessionnel de la BID (FSO, Fund for Special Operations) se posera à nouveau. En effet, les négociations relatives à la neuvième reconstitution du FSO sont retardées. Pour permettre à la BID de maintenir ses prêts pour les pays les plus pauvres de la région et pour contribuer financièrement à l'initiative pour la réduction de la dette de la Bolivie et de la Guyane, un accord de 1,2 milliard de dollars a été conclu pour 1998-1999 en puisant encore dans les ressources du fonds.

\section{8. «L'ACTION DE LA SUISSE POUR LES PEUPLES AUTOCHTONES»}

Suite à des échanges entre, d'une part, la Direction du développement et de la coopération (DDC) et la Direction politique IV du DFAE, chargée de la politique des droits de l'homme et de la politique humanitaire (DPIV), et, d'autre part, la Coordination des ONG pour les peuples autochtones ${ }^{26}$, la DDC et la Direction politique du DFAE ont publié, en juin 1999, un rapport sur l'action de la Suisse pour les peuples autochtones ${ }^{27}$.

Les peuples autochtones y sont définis comme ceux qui s'autodéfinissent comme autochtones et qui fondent leurs revendications sur l'antériorité de leur occupation du territoire par rapport aux sociétés dominantes; sur le fait que le lien à la terre est consubstantiel à leur existence et sur leur position marginalisée du point de vue de leurs droits. En outre, tous ces peuples conservent en général des systèmes économiques adaptés à leur environnement et des systèmes d'organisation sociale qui leur sont propres.

26. La Coordination des ONG pour les peuples autochtones réunit les organisations suivantes: Société pour les peuples menacés; Centre de documentation, de recherche et d'information des peuples autochtones (doCip); Incomindios; Traditions pour Demain; Institut für Ökologie und Aktion-Ethnologie (infoe Schweiz); Fonds Bruno Manser; Indigenous Affairs International Working Group (IWGIA); Nouvelle Planète.

27. DDC, L'action de la Suisse pour les peuples autochtones, DDC, Berne, 1999. 


\section{$\square$ Position de la Suisse à l'égard des peuples autochtones}

Depuis une dizaine d'années, les revendications des peuples autochtones sont prises en considération au niveau international, et le savoir de ces peuples est de plus en plus reconnu comme facteur d'un développement économique, collectif et durable. De même, la coopération suisse, tout en adhérant au principe de l'universalité des droits de l'homme, reconnaît que les différences culturelles doivent être prises en considération. En ce qui concerne les peuples autochtones, la Suisse intervient aussi bien sur le plan bilatéral que multilatéral. Elle apporte également son financement à plusieurs ONG suisses de développement qui œuvrent dans ce domaine.

Les peuples autochtones sont souvent victimes de violations des droits de l'homme (discrimination, réinstallations forcées, assimilation culturelle, génocide...). Par ailleurs, le développement économique des dernières décennies affecte gravement leur système culturel, qui est d'une grande importance notamment pour la sauvegarde de l'environnement, tant aux niveaux local que régional et global. Il en résulte une menace sérieuse pour l'existence même de ces peuples et une atteinte grave à l'écosystème et à l'environnement de la région.

\section{"L'action de la Suisse en faveur des peuples autochtones» en bref}

Sur le plan bilatéral

- Observation et analyse de la situation.

- Préparation de visites de responsables gouvernementaux. Prises de position qui permettent d'aborder cette question au niveau de la discussion entre gouvernements.

- Contacts avec les ONG de défense des droits des peuples autochtones.

Sur le plan multilatéral

- Participation au Groupe de travail sur les peuples autochtones et sur le projet de déclaration de I'ONU.

a Prises de position en faveur de la promotion des droits des peuples autochtones au niveau des enceintes internationales (Commission des droits de I'homme, CNUED, Sommet social de Copenhague, FIDA, OMC, Convention sur la biodiversité).

La Convention n 169 de l'0IT "concernant les peuples indigènes et tribaux dans les pays indépendants" n'a pas encore été ratifiée par la Suisse, mais le Conseil fédéral en approuve les objectifs*. Seuls 13 pays ont pour l'instant ratifié cette convention, dont 9 pays latino-américains (Bolivie, Colombie, Costa Rica, Equateur, Guatemala, Honduras, Mexique, Paraguay et Pérou) et trois pays industrialisés (Danemark, Norvège et Pays-Bas).

Source: DDC, L'action de la Suisse pour les peuples autochtones, 1999

* Le texte de cette convention figure sur le site Internet http://ilolex.ilo.ch:1567/public/french/50normes/infleg/ilofre/index.htm.

Deux des principaux objectifs définis dans le Rapport sur la politique extérieure de la Suisse dans les années 90 ainsi que dans le Rapport sur les relations NordSud sont la promotion des droits de l'homme, de l'Etat de droit et des processus démocratiques ainsi que la préservation du milieu naturel. Dans ce contexte, la promotion des droits des peuples autochtones apparaît comme un thème qui s'inscrit parfaitement dans les préoccupations de la Suisse: la défense de ces peuples est non seulement indispensable à leur propre survie, mais participe également à la paix, à la sécurité et à la prévention des conflits. Ainsi la Suisse se 
déclare-t-elle en faveur de l'adoption par l'ONU du projet de déclaration sur les droits des peuples autochtones et d'une participation de ces derniers aux processus décisionnels les concernant afin de définir et de défendre davantage leurs droits.

En outre, toujours en ce qui concerne les droits des autochtones, la Suisse est en faveur de la mise en œuvre du principe de subsidiarité (droit à l'autonomie et droit de s'administrer de façon autonome au sein d'un Etat) par opposition à l'isolation ou à l'assimilation forcée. Parallèlement à l'octroi d'une plus grande participation politique, sociale et économique aux autochtones au niveau national, la Suisse plaide pour la création d'un forum permanent au Conseil économique et social des Nations Unies (ECOSOC) afin de permettre aux autochtones de faire valoir leurs droits au niveau international. Finalement, la Suisse estime qu'il convient de mettre en valeur les savoirs traditionnels de ces peuples, de préserver leur patrimoine culturel et de prendre en compte les préoccupations des autochtones pour la protection de leur propriété intellectuelle.

\section{$\square$ Position de la coopération suisse} au développement à l'égard des peuples autochtones

Dans le cadre plus spécifique de sa coopération au développement, la Suisse a pour principaux objectifs le développement humain, la lutte contre la pauvreté, la promotion des droits de l'homme, le développement équilibré hommesfemmes, le renforcement du pouvoir économique, social, politique et culturel des populations les plus désavantagées, ainsi que la promotion d'un développement durable et d'une croissance économique respectueuse des ressources naturelles. Ainsi, les peuples autochtones apparaissent comme des partenaires privilégiés de la coopération suisse.

\section{Principes généraux}

En ce qui concerne la promotion des droits des peuples autochtones, la coopération suisse se base sur ses lignes directrices Promotion des droits de l'homme et coopération au développement. Par conséquent, les projets concernant les peuples autochtones devront être examinés afin de pouvoir évaluer dans quelle mesure ils risqueraient de porter atteinte aux droits de l'homme. Les principes préconisant le recours à des mesures positives et le dialogue politique seront également privilégiés.

En outre, dans le cadre de ses projets et programmes, la DDC entend privilégier la recherche de solutions spécifiques et différenciées selon les contextes économiques, sociaux et politiques tout en mettant l'accent sur le dialogue entre peuples autochtones au travers de leurs organisations et sur leur participation à la définition de ces projets et programmes.

\section{Recommandations}

Plus concrètement, dans le cadre de la promotion des droits des populations autochtones, la coopération suisse dirige ses activités dans les domaines suivants: promotion du développement participatif (empowerment) et renforcement des capacités; promotion de l'Etat de droit (good governance); lutte contre la pauvreté; promotion des droits culturels et préservation de l'identité des peuples autochtones; promotion d'un développement durable et prise en compte des intérêts des populations les plus désavantagées dans le cadre des règles commerciales internationales (Accord sur les aspects des droits de propriété intellectuelle liés au commerce - ADPIC). 
Afin de mettre ces priorités en pratique, le rapport sur l'action de la Suisse pour les peuples autochtones recommande la prise en considération des principes suivants dans les activités de la DDC:

- renforcer les compétences (notamment en matière de représentation) des peuples autochtones et mettre en valeur l'importance des organisations autochtones;

- renforcer les capacités gouvernementales et institutionnelles, et soutenir les efforts portant sur l'amélioration et l'application de la législation;

- renforcer le pouvoir économique social et politique des populations les plus pauvres (dont les peuples autochtones);

- accorder une attention particulière aux systèmes autochtones d'organisation, d'éducation et de santé qui seront mis en valeur en collaboration avec les communauté concernées;

- soutenir l'appui aux processus de décentralisation et de délégation du pouvoir décisionnel au niveau local en veillant à ce que les intérêts des autochtones soient pris en considération.

Dans le cadre de la promotion d'un développement durable, il s'agira de prendre en compte et de préserver les connaissances traditionnelles de gestion de l'environnement et d'utilisation durable des ressources naturelles des autochtones (biodiversité). En outre, 1'amélioration des conditions-cadres dans le domaine des ressources génétiques doit demeurer une priorité de la DDC.

Finalement, le souci de prendre en considération les intérêts, les besoins, les potentialités et la situation particulière des autochtones, ainsi que leur implication dans les différentes phases des projets (planification, conduite, évaluation), s'inscrit en filigrane dans toutes les interventions de la DDC pour la promotion des droits des peuples autochtones.

\section{$\square$ Réaction des $O N G$ de défense des droits des peuples autochtones}

La Coordination des ONG approuve le contenu du rapport de la DDC, reconnaît les efforts du DFAE pour la promotion des peuples autochtones, et encourage la Suisse à soutenir les efforts des peuples autochtones pour que leurs droits soient reconnus et garantis, en particulier par la ratification de la Convention n ${ }^{\circ} 169$ de l'OIT.

\subsection{PARTENARIATS SCIENTIFIQUES AVEC LES PAYS EN DÉVELOPPEMENT}

\section{$\square K F P E$}

La recherche scientifique fait partie intégrante du développement car elle contribue à résoudre les problèmes les plus lancinants aux niveaux tant international que local. A cet effet, il est nécessaire de mettre en place et de renforcer les capacités de recherche, en particulier dans les pays économiquement défavorisés. C'est la tâche que s'est donnée la $\mathrm{KFPE}^{28}$. A l'heure actuelle, on encourage

28. La Commission suisse pour le partenariat scientifique avec les pays en développement (Kommission für Forschungspartnerschaften mit Entwicklungsländern, KFPE) s'est dotée de nouveaux statuts en 1999 et est ainsi devenue une commission de l'organisme qui l'a créée, c'est-à-dire la Conférence des académies scientifiques suisses (CASS). La KFPE a pour principal objectif de promouvoir le partenariat scientifique pour contribuer à un développement durable dans le monde entier. Le professeur T.A. Freyvogel (Institut tropical suisse, Bâle), qui est le maître d'œuvre de la création de la commission et qui a présidé à sa destinée depuis sa fondation en 1994, s'est retiré à la fin de mars 1999. Il a été remplacé à la tête de la commission par le professeur Hans Hurni, codirecteur du Centre de développement et d'environnement (Center for Development and Environment, CDE) de l'Institut géographique de l'Université de Berne. 
encore peu les partenariats scientifiques avec les pays en développement. De plus, ces partenariats ne sont guère reconnus, même sur le plan international, bien que des milieux toujours plus larges préconisent la promotion de la recherche pour résoudre les problèmes des pays du $\mathrm{Sud}^{29}$. Cette recherche doit bien entendu s'orienter avant tout vers les besoins et les priorités des pays en développement et de leurs communautés locales.

La KFPE s'est récemment associée avec les institutions suisses qui possèdent une longue expérience du partenariat scientifique avec les pays en développement. Cette nouvelle alliance accroît le flux d'informations spécialisées, améliore leur application par les chercheurs du Nord et du Sud et fédère les forces en présence pour mener des actions communes. La KFPE s'est aussi plus largement ouverte aux milieux intéressés pour susciter le dialogue avec les partenaires les plus variés. Ceux-ci proviennent de la recherche, de l'administration, de la coopération au développement, de l'économie et de diverses fondations ${ }^{30}$. Cette ouverture a pour objectif de mieux exploiter les partenariats scientifiques et de promouvoir leur reconnaissance.

\section{$\square$ Programme prioritaire environnement}

A la fin de 1999, les projets du module 7 «Développement et environnement» du Programme prioritaire environnement (PPE) sont entrés dans leur phase finale ${ }^{31}$. Au nombre de 14, ces projets, qui avaient pour objectif d'appliquer dans la pratique les principes du partenariat scientifique, ont été répartis dans trois groupes: trois projets portent sur la gestion des ressources naturelles, six sur la gestion de l'environnement urbain et cinq sur les stratégies et sur la gestion des conflits ${ }^{32}$. Dans l'ensemble, la Suisse se montre plus sensible aux problèmes du développement à l'échelle mondiale, mais beaucoup reste à faire dans ce domaine. Le système de référence international que l'on utilise aujourd'hui pour évaluer les résultats de la recherche se fonde encore trop sur les institutions et sur les thèmes des pays développés, de sorte qu'il ne tient pas assez compte de critères tels que la pertinence pour la politique de développement, la concrétisation et la promotion des capacités. L'évaluation du module «Développement et environnement» a par ailleurs montré que les projets sont de trop courte durée pour assurer une promotion des capacités efficace et de l'envergure souhaitée. Le module a toutefois contribué à faire accepter les partenariats scientifiques avec des pays économiquement défavorisés et à mieux les faire reconnaître en Suisse.

\section{Nouvelles initiatives}

Les initiatives suivantes prouvent que les partenariats scientifiques suscitent un intérêt croissant:

๖ à la Conférence mondiale sur la science, organisée par l'UNESCO et le CIUS (Conseil international des unions scientifiques) à Budapest en juillet 1999, la

29. Banque mondiale, Rapport sur le développement dans le monde 1998-1999. Le savoir au service du développement, Washington, 1999; Commission européenne, Scientific Partnership for Development, Bruxelles, 1998, 56 p.; J. Sachs, «Helping the World's Poorest», The Economist, 14.8.99, pp. 17-20.

30. La liste des membres et des institutions associées figure sur le site Internet de la KFPE, page www.kfpe.unibe.ch/ members.html.

31. Le président de la KFPE commente le module 7 dans un numéro spécial que Panorama a consacré à ce module (décembre 1999) et qui peut être obtenu auprès de la direction du PPE, Länggassstrasse 23, 3012 Berne.

32. Un résumé des projets figure sur le site Internet de la KFPE, page www.kfpe.unibe.ch/chrespro.html. 
délégation suisse, emmenée par le secrétaire d'Etat Charles Kleiber, a présenté les partenariats scientifiques avec les pays en développement comme étant l'un des thèmes auxquels la Suisse compte s'atteler à l'avenir. Le Guide du partenariat scientifique avec des pays en développement: 11 principes de la KFPE a également été présenté à cette conférence ${ }^{33}$;

- prenant le module «Développement et environnement» pour modèle, le Fonds national suisse (FNS) et la DDC ont convenu d'un programme commun destiné à soutenir des projets basés sur le partenariat. Les deux institutions consacreront dans un premier temps 1 million de francs par an à ce programme. Non seulement les projets concernés devront remplir des critères scientifiques, mais ils seront aussi évalués sur la base de leur pertinence pour le développement et de leur contribution à la promotion des capacités dans le pays partenaire. Le FNS et la DDC sélectionneront ensemble les projets bénéficiaires ${ }^{34}$, dont la description devra également préciser dans quelle mesure ils respectent les 11 principes du guide de la KFPE;

- un programme interne de la DDC est destiné à soutenir des jeunes chercheurs de Suisse ou de pays en développement, qui souhaitent mener des projets fondés sur le partenariat. Dans le cadre de ces projets, on met également l'accent sur la concrétisation des résultats. Un autre programme de la DDC favorise les échanges interuniversitaires entre la Suisse et les pays du Sud et de l'Est ${ }^{35}$;

๖ un nouveau programme de la DDC (1 million de francs pour trois ans) appuie les projets menés en collaboration avec des partenaires du Sud au CFRC (Centre pour la formation postgrade, les relations internationales et la coopération, Ecole polytechnique fédérale, Lausanne);

- le FNS poursuivra son programme de coopération scientifique dans le cadre du programme pour l'Europe de l'Est du DFAE. Ce programme, qui met l'accent sur les partenariats entre des institutions suisses et d'Europe de l'Est, vise avant tout à renforcer les institutions partenaires à l'Est ${ }^{36}$;

- le FNS envisage d'une part d'accorder une aide initiale à la création d'une organisation autonome de promotion de la recherche dans un pays de son choix, et d'autre part souhaite désormais aussi conclure des accords classiques de coopération visant à encourager les échanges scientifiques avec les organisations de promotion de la recherche des pays en développement.

Relevons toutefois qu'outre les quelques instruments brièvement présentés cidessus, la Suisse compte divers instituts scientifiques qui possèdent une longue expérience du partenariat avec le Sud et/ou l'Est et qu'ils sont très actifs dans ce domaine $^{37}$.

Après la Conférence mondiale sur la science mentionnée ci-dessus, le secrétaire d'Etat Charles Kleiber a déclaré que la Suisse se doit absolument de coopérer avec les pays en développement. C'est pourquoi au moins un des pôles de

33. Ce guide, publié en six langues, peut être obtenu gratuitement auprès du secrétariat de la KFPE, Bärenplatz 2, 3011 Berne, ou téléchargé à partir du site Internet de la KFPE www.kfpe.unibe.ch

34. Pour plus d'informations, voir le site Internet du FNS, page www.snf.ch/Interframeset_f.html.

35. Voir, sur le site Internet de la KFPE, les pages respectives des deux projets: www.kfpe.unibe.ch/chrespro.html\#jc et www.kfpe.unibe.ch/chrespro.html\#echu.

36. Voir la note 34 ci-dessus.

37. La plupart de ces instituts figurent sur le site Internet de la KFPE. 
recherche nationaux (PRN) sera consacré à la coopération Nord-Sud ${ }^{38}$. Ce nouvel instrument d'encouragement de la recherche du FNS débutera dès 2001 et devrait s'étendre sur dix ans. En collaboration avec la KFPE, diverses institutions possédant une longue expérience de la coopération scientifique ont présenté un avant-projet de PRN commun, qui a pour titre «NCCR North-South: Research Partnerships for Mitigating Syndromes of Global Change». Le programme devrait être subdivisé en huit projets partiels qui se fonderont sur le partenariat scientifique pour étudier divers syndromes ${ }^{39}$ de l'évolution mondiale: zones urbaines et périurbaines, régions semi-arides et régions de plaine et de hauts plateaux. Contrairement à ce qui est prévu pour les PRN, le projet NordSud n'aura pas une leading house rattachée à une seule université, mais représentera, conformément à son objectif, la «haute école suisse» dans les pays du Sud et de l'Est. Cette structure s'est imposée d'emblée, car toutes les hautes écoles participant au projet accordent une très petite place à la coopération scientifique avec ces pays. Par ailleurs, pratiquement chaque université ou haute école compte plusieurs institutions possédant de grandes compétences dans ce domaine. La DDC a pris une part active à l'élaboration de l'avant-projet et assuré un soutien efficace aux futurs partenaires des pays en développement. 


\section{SOURCES}

Evolution générale de l'aide publique au développement des pays du CAD

OCDE, Coopération pour le développement. Rapport 1998: Efforts et politiques des membres du Comité d'aide au développement, OCDE, Paris, 1999.

OCDE, «Les apports financiers aux pays en développement en 1998: augmentation de l'aide; diminution prononcée des apports privés», Communiqué de presse, 10.6.99.

Aide publique fournie par la Suisse

«Message du Conseil fédéral concernant la continuation de la coopération technique et de l'aide financière en faveur des pays en développement» du 7 décembre 1998, Feuille fédérale (message n 98.072).

DDC et OFAEE, Coopération internationale de la Suisse. Rapport annuel 1998, trois brochures: «Coopération au développement», «Aide humanitaire», «Coopération avec l'Europe de l'Est», Berne, 1999.

DDC, Promotion des droits de l'homme et coopération au développement. Lignes directrices, DDC, Berne, 1997, 24 p.

DDC, Lutte contre la corruption. Lignes directrices, DDC, Berne, 1998, 24 p.

DDC, L'action de la Suisse pour les peuples autochtones, DDC, Berne, 1999, 32 p.

DDC, Quick Money - Easy Money? A Report on Child Labour in Tourism, DDC, Working Paper 4/99, August 1999, $110 \mathrm{p}$.

R. Pasquier, La bonne gouvernance, le développement participatif et la réduction de la pauvreté rurale, document de travail de la DDC 5/99, DDC, Berne, $24 \mathrm{p}$.

DDC, Politique de la DDC pour le développement social, document de travail de la DDC 9/99, DDC, Berne, 28 p.

DDC et OFAEE, Si les pauvres sont pauvres, c'est de leur faute. Questions et réponses au sujet de la coopération internationale de la Suisse (argumentaire sur la coopération au développement), DDC et OFAEE, Berne, 1999, 36 p.

DDC, Service de presse, parution 4 fois par an. Thèmes récents: villes du Sud, 20.12.99; Club du Sahel, 30.8.99; coopération au développement et politique de migration, 30.8.99.

DDC, Agenda politique, parution mensuelle.

DDC, Newsletter (informations en bref), 6 numéros par an.

DDC, Un Seul Monde (revue), parution 4 fois par an.

DDC, Image directrice de la DDC, Berne, 1999.

DFAE, La Suisse et le Monde (revue), parution 4 fois par an. Commande: tél. 031/322.31.53.

Neue Zürcher Zeitung, «Neue Schweizer Entwicklungsansätze in Indien», 8.12.98.

\section{SITES INTERNET, ADRESSES ET COMMANDE DE PUBLICATIONS}

$\mathrm{CAD}$ (OCDE): www.oecd.org/dac. Ce site permet de se connecter à ceux des services de coopération des pays membres du CAD.

Corps suisse d'aide en cas de catastrophe: www.skh.ch.

DDC: www.ddc-admin.ch.

DFAE: www.dfae.admin.ch. Ce site contient tout un chapitre sur les organisations internationales: www.dfae.admin.ch/site/f/aussenpolitik/internationale.html.

Euforic, Forum européen de coopération internationale: www.oneworld.org/euforic/index.html

FNS: Wildhainweg 20, 3001 Berne; www.snf.ch.

KFPE, secrétariat: Bärenplatz 2, 3011 Berne; www.kfpe.unibe.ch.

Seco: www.seco.admin.ch

Commande des publications de la DDC: DDC, Médias et communication, 3003 Berne, fax 031/324.13.48, e-mail: info@deza.admin.ch. 